\title{
High-Order Local Maximum Principle Preserving (MPP) Discontinuous Galerkin Finite Element Method for the Transport Equation
}

\author{
R. Anderson, V. Dobrev, Tz. Kolev, D. Kuzmin, M. Quezada de Luna, R. Rieben and V. Tomov
}

\begin{abstract}
In this work we present a FCT-like Maximum-Principle Preserving (MPP) method to solve the transport equation. We use high-order polynomial spaces; in particular, we consider up to 5th order spaces in two and three dimensions and 23rd order spaces in one dimension. The method combines the concepts of positive basis functions for discontinuous Galerkin finite element spatial discretization, locally defined solution bounds, element-based flux correction, and non-linear local mass redistribution. We consider a simple 1D problem with non-smooth initial data to explain and understand the behavior of different parts of the method. Convergence tests in space indicate that high-order accuracy is achieved. Numerical results from several benchmarks in two and three dimensions are also reported.
\end{abstract}

\section{Introduction}

We are interested in Maximum-Principle Preserving (MPP) high-order finite element discontinuous Galerkin (DG) discretizations of the transport equation. It is known that the high polynomial degree nature of such discretizations is prone to monotonicity violations. While small oscillations could be accepted in some cases, in many applications they can lead to unphysical values, e.g., values outside of $[0,1]$ should not occur for material volume fractions in the context of multi-material simulations.

The approach considered in this paper is based on flux limiting. Flux limiting methods utilize a low- and a high-order solution selectively. The idea is to use the high-order solution when the solution is smooth and switch to the low-order method near discontinuities. This is usually done via nonlinear limiting factors computed based on the solution. A popular flux limiting method is the Flux Corrected Transport (FCT) introduced for a one dimensional linear problem using finite differences in [6]. Later in [27] the methodology is presented in a more generalized format for multiple dimensions and considering nonlinear problems. An extensive and detailed description of this method can be found in [16]. A similar class of methods in [10,11], known as hybrid self-adjusting schemes, automatically and smoothly switches between a first- and a second-order solution depending on the steepness of the solution. The method uses an "automatic switch" parameter to smoothly and automatically switch between the two methods. In [9] the FCT method is used based on a second-order continuous Galerkin scheme to obtain a second-order locally maximum principle preserving method. 
This study is motivated by the previous work for MPP high-order finite element ALE remap [4]. While the FCT method presented in [4] (denoted by DG-FCT throughout this manuscript) is formally MPP and satisfactory up to $\mathbb{Q}_{3}$ finite elements, using higher order polynomial spaces leads to oscillations as the ones shown in Figure 1. The main cause of these oscillations is that the sparsity pattern of the DG-FCT advection matrix includes a high number of degrees of freedom, causing high variations in the definition of maximum and minimum admissible values. It is clear that special effort is needed to address the case of higher order (greater than $\mathbb{Q}_{3}$ ) polynomial spaces.

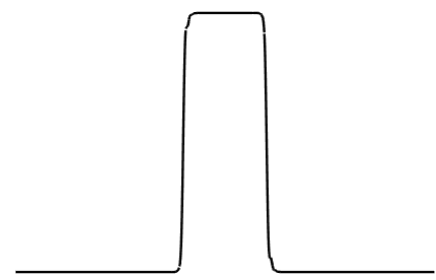

(a) $\mathbb{Q}_{3}$ finite elements

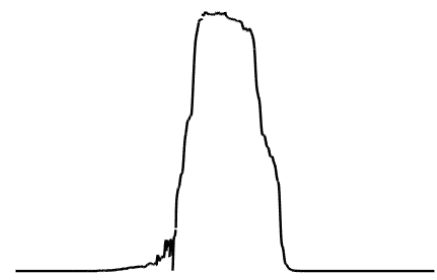

(b) $\mathbb{Q}_{5}$ finite elements

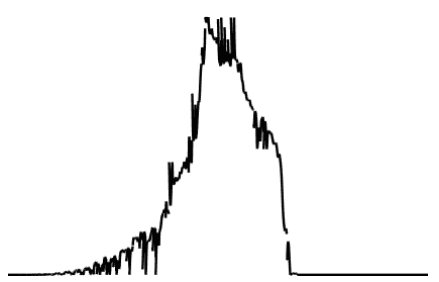

(c) $\mathbb{Q}_{7}$ finite elements

Figure 1: Example of the problematic behavior of the DG-FCT method on a 1D advection problem with refinement in polynomial order. All solutions are within their original $[0,1]$ bounds.

In this paper we address the aforementioned issue by presenting a new FCT-like MPP method that is applicable to both advection remap and the transport equation. After reviewing the underlying DG finite element discretization and the DG-FCT method, we introduce the concept of localized stencils that define tighter bounds for each degree of freedom. This is combined with an element-based FCT formulation (denoted DG-EFCT). In DG-EFCT, instead of adjusting all incoming fluxes, one adjusts the value at each degree of freedom, causing a mass error in each cell. These mass errors are corrected by a sub-zonal mass redistribution process that results in a non-linear problem to be solved in each cell. We show that the DG-EFCT method is capable of reducing the type of oscillations shown in Figure 1, while still achieving the convergence rates of the DG-FCT method reported in [4] for smooth profiles, i.e., order $p+1$ in monotone regions and rates of at most $2-2.5$ around local extrema.

As in [4], our high-order finite element shape functions are represented by the Bernstein-Bézier polynomials [3]. This approach has been employed in past studies as well, for example [2], where the authors demonstrate that these polynomials can be employed in a way that provides both (i) local monotonicity preservation and (ii) accuracy above second order. In this paper we follow the same path. Furthermore, we show that, in our particular DG framework, the use of the Bernstein-Bézier polynomials yields a better low-order method than the traditional Gauss-Legendre or Gauss-Lobatto bases.

Another popular approach to impose maximum principle preservation is [28], where the authors propose a limiting process to obtain arbitrary high-order methods for multiple dimensions for scalar conservation laws. The authors show that to do this it is sufficient to compute the limiters by evaluating a polynomial over a set of quadrature points. Therefore, the extension to arbitrary order and multiple dimensions is trivial. This polynomial can be reconstructed if WENO methods are considered 
or it is directly obtained for DG schemes. Using this idea, in [29], positivity preserving high-order methods are proposed based on discontinuous Galerkin methods to solve the compressible Euler equations on rectangular meshes. These ideas are later extended to triangular meshes in [30]. To do this the authors propose a specific quadrature rule. The methodology in [28] is adapted to obtain secondorder [31] and more than second-order [24] strictly maximum principle preserving methods based on discontinuous Galerkin schemes to solve convection-diffusion equations. Using similar ideas but limiting the numerical fluxes directly instead of the (reconstructed) polynomial and using finite differences and finite volumes, the authors in [25] propose a parametrized flux limiter for one dimensional scalar conservation laws to obtain strictly (or global) maximum principle preserving solutions. Moreover, using finite volume methods of up to 4th order, the authors prove that high order accuracy is preserved for smooth solutions. The method is later extended to two dimensions in [21] and to convected dominated problems in [26]. Our understanding of the above methods is that they enforce global maximum principle, which is significantly different from the local MPP property we are considering in this paper. Oscillations, as the ones shown on Figure 1, do not violate the global bounds of the solution, however, they are clearly undesirable.

The remainder of this manuscript has the following organization. In Section 2 we review the basic transport equation we are interested in solving as well the general high-order DG formulation (which is expected to be non-MPP). Low order (or 1st order) MPP methods are the foundations for any non-linear, high-order FCT method and in Section 3 we show that the choice of the Bernstein basis functions in the DG formulation has a large effect on the quality of the low-order MPP method. Review of the DG-FCT approach in the context of high-order DG, followed by description of the main challenge this paper addresses, is presented in Section 4. In Section 5 we present a "localized" DG-FCT method which uses a reduced order stencil for computing bounds. In Section 6 we review the DG-EFCT approach. Unlike DG-FCT, DG-EFCT does not naturally conserve mass, so an additional correction step is required. We describe two approaches for recovering mass conservation, one based on a uniform (element-wise) flux rescaling (DG-EFCT-U) and one based on a non-linear mass redistribution solve (DG-EFCT-N). Section 7 describes how the new methods can be used for the purposes of ALE remap, where the velocity field is interpreted as the mesh displacement field. Finally, in Section 8 we present numerical results on a set 2D and 3D benchmark problems.

\section{Preliminaries}

We consider the transport equation given by

$$
\begin{aligned}
\partial_{t} u(\mathbf{x}, t) & =\nabla \cdot(\mathbf{v}(\mathbf{x}, t) u(\mathbf{x}, t)), & \forall(\mathbf{x}, t) \in \Omega \times[0, T], \\
u(\mathbf{x}, t=0) & =u_{0}(\mathbf{x}), & \forall \mathbf{x} \in \Omega,
\end{aligned}
$$

where $\Omega \subset \mathbb{R}^{d}$ is an open domain, $0<T \in \mathbb{R}$ is the final time, $u: \Omega \times[0, T] \rightarrow \mathbb{R}$ is the transported solution, $\mathbf{v}: \Omega \times[0, T] \rightarrow \mathbb{R}^{d}$ is divergence-free velocity field with $d \in\{1,2,3\}$ being the spatial dimension, and $u_{0}: \Omega \rightarrow \mathbb{R}$ is the initial condition. In this study we consider periodic boundary conditions for $1 \mathrm{D}$ problems, and $\mathbf{v}(\mathbf{x} \in \partial \Omega) \cdot \mathbf{n}=0$ for $2 \mathrm{D}$ and $3 \mathrm{D}$ problems. 
It is our aim to obtain a solution that preserves the maximum principle locally. Given a finite element solution $u_{h}^{n}=\sum_{i} \phi_{i} U_{i}^{n}$ at time $t=t_{n}$, the solution $u_{h}^{n+1}=\sum_{i} \phi_{i} U_{i}^{n+1}$ of a MaximumPrinciple Preserving (MPP) method is said to satisfy the discrete maximum principle locally if

$$
\min _{j \in N_{i}} U_{j}^{n}=: U_{i}^{\min } \leq U_{i}^{n+1} \leq U_{i}^{\max }:=\max _{j \in N_{i}} U_{j}^{n}, \forall i
$$

where $N_{i}$ defines some neighborhood of the $i$-th degree of freedom (DOF). Conventionally, $N_{i}$ is defined through the sparsity pattern of the discrete advection operator. If continuous Galerkin finite elements are used, this sparsity pattern is given by the support of the $i$-th shape function, $\phi_{i}$. With discontinuous Galerkin finite elements, this sparsity pattern consists of all DOFs on the given cell and on adjacent cells sharing a face with it.

\subsection{High-Order DG Spatial Discretization}

Consider a computational mesh $\mathcal{T}_{h}$ with internal faces $\mathcal{F}_{h}$. We define the discontinuous finite dimensional space $X_{h}=\left\{\phi(\mathbf{x}) \in L^{2}(\Omega):\left.\left.\phi\right|_{K} \in \mathbb{Q}\right|_{K}, \forall K \in \mathcal{T}_{h}\right\}$ where $\left.\mathbb{Q}\right|_{K}$ is a polynomial space over the element $K$. Let $\left\{\phi_{1}, \ldots, \phi_{N}\right\}$ be a basis of $X_{h}$, where $N=\operatorname{dim}\left(X_{h}\right)$, such that $\sum_{i}^{N} \phi_{i}(\mathbf{x})=1$.

Consider the transport equation (1), multiply it by $\phi \in X_{h}$, integrate over $\Omega$ and integrate by parts the advection term to obtain

$$
\int_{\Omega}\left(\partial_{t} u\right) \phi d \mathbf{x}=-\sum_{K \in \mathcal{T}_{h}} \int_{K} u(\mathbf{v} \cdot \nabla \phi) d \mathbf{x}+\sum_{f \in \mathcal{F}_{h}} \int_{f} u\left(\mathbf{v} \cdot \mathbf{n}_{f}\right) \phi d \mathbf{s},
$$

where $\mathbf{s} \in \mathbb{R}^{d-1}$ and $\mathbf{n}_{f}$ is the unit normal vector at face $f$. Let $u_{h} \in X_{h}$ be the finite element approximation of $u$. Since $u_{h}$ is discontinuous across $f$ we can't replace $u$ by $u_{h}$ in (3) or we would obtain multiple values over $f$. Therefore, we define numerical fluxes associated with the internal faces to get

$$
\int_{\Omega}\left(\partial_{t} u_{h}\right) \phi d \mathbf{x}=-\sum_{K \in \mathcal{T}_{h}} \int_{K} u_{h}(\mathbf{v} \cdot \nabla \phi) d \mathbf{x}+\sum_{f \in \mathcal{F}_{h}} \int_{f}\left\{u_{h} \mathbf{v} \cdot \mathbf{n}_{f}\right\}_{*}[[\phi]] d \mathbf{s},
$$

where $[[\phi]]:=\phi^{-}-\phi^{+}, \phi^{ \pm}(\mathbf{x})=\lim _{\xi \rightarrow 0^{+}} \phi\left(\mathbf{x} \pm \xi \mathbf{n}_{f}(\mathbf{x})\right)$ and

$$
\left\{u_{h} \mathbf{v} \cdot \mathbf{n}_{f}\right\}_{*}=\left(\mathbf{v} \cdot \mathbf{n}_{f}\right)\left(\frac{\left.u_{h}\right|_{K_{1}}+\left.u_{h}\right|_{K_{2}}}{2}\right)-\frac{1}{2}\left|\mathbf{v} \cdot \mathbf{n}_{f}\right|\left[\left[u_{h}\right]\right],
$$

which is known as Godunov (upwind) flux [20,23]. Method (4) can be recast in matrix-vector form as

$$
M \frac{d U^{H}(t)}{d t}=K U^{H}(t)
$$


where $U^{H}(t)=\left[U_{1}^{H}(t), \ldots, U^{H}(t)_{N}\right]^{t}$ are the DOFs of the finite element solution $u_{h}(\mathbf{x}, t)$ at time $t$ and $M$ and $K$ are the mass and transport matrices whose $i j$-th elements are given by:

$$
\begin{aligned}
M_{i j} & =\int_{\Omega} \phi_{i} \phi_{j} d \mathbf{x} \\
K_{i j} & =-\int_{\Omega} \phi_{j}\left(\mathbf{v} \cdot \nabla \phi_{i}\right) d \mathbf{x}+\sum_{f \in \mathcal{F}_{h}} \int_{f}\left\{\phi_{j} \mathbf{v} \cdot \mathbf{n}_{f}\right\}_{*}\left[\left[\phi_{i}\right]\right] d \mathbf{s} .
\end{aligned}
$$

The method (5) is mass conservative in the following sense:

$$
\int_{\Omega} u_{h}(\mathbf{x}, t) d \mathbf{x}=\int_{\Omega} u_{h}(\mathbf{x}, t=0) d \mathbf{x} \Longleftrightarrow \sum_{j} U_{j}^{H}(t) m_{j}=\sum_{j} U_{j}^{H}(t=0) m_{j},
$$

where $m_{j}=\sum_{i} \int_{\Omega} \phi_{i} \phi_{j} d \mathbf{x}=\int_{\Omega} \phi_{j} d \mathbf{x}$. To see this consider the row sum of (5),

$$
\begin{aligned}
\sum_{i} \sum_{j} M_{i j}\left(\frac{d U_{j}^{H}(t)}{d t}\right)=\sum_{i} \sum_{j} K_{i j} U_{j}^{H}(t) & \Longrightarrow \sum_{j} m_{j}\left(\frac{d U_{j}^{H}(t)}{d t}\right)=\sum_{j} U_{j}^{H}(t) \sum_{i} K_{i j}=0 \\
& \Longrightarrow \sum_{j} U_{j}^{H}(t) m_{j}=\sum_{j} U_{j}^{H}(t=0) m_{j},
\end{aligned}
$$

where $K$ has zero column sums since

$$
\sum_{i} K_{i j}=-\int_{\Omega} \phi_{j}\left[\mathbf{v} \cdot \nabla\left(\sum_{i} \phi_{i}\right)\right] d \mathbf{x}+\sum_{f \in \mathcal{F}_{h}} \int_{f}\left\{\phi_{j} \mathbf{v} \cdot \mathbf{n}_{f}\right\}_{*}\left[\left[\sum_{i} \phi_{i}\right]\right] d \mathbf{s}=0
$$

by partition of unity, i.e., $\sum_{i} \phi_{i}=1$. Because $\nabla \cdot \mathbf{v}=0$ is assumed, the row sums of $K$ are also zero:

$$
\sum_{j} K_{i j}=-\int_{\Omega} \mathbf{v} \cdot \nabla \phi_{i} d \mathbf{x}+\sum_{f \in \mathcal{F}_{h}} \int_{f} \mathbf{v} \cdot \mathbf{n}_{f}\left[\left[\phi_{i}\right]\right] d \mathbf{s}=-\int_{\mathcal{K}} \mathbf{v} \cdot \nabla \phi_{i} d \mathbf{x}+\int_{\partial \mathcal{K}} \mathbf{v} \cdot \mathbf{n} \phi_{i},
$$

where $\mathcal{K}$ is the cell where $\phi_{i}$ is supported. Assuming that the integrals are computed exactly, the row sum is equal to the integral over $\mathcal{K}$ of $\left(\phi_{i} \nabla \cdot \mathbf{v}\right)=0$.

\subsection{Time Discretization}

For simplicity we consider Forward Euler integration in time. However, we extend the results to highorder approximations via Strong Stability Preserving (SSP) methods [8]. Moreover, all numerical experiments, unless otherwise noted, are performed via a third order (three stage) Runge-Kutta SSP method. The time discretization of (5) via Forward Euler is given by:

$$
M\left(\frac{U^{H}-U^{n}}{\Delta t}\right)=K U^{n}
$$

where $U^{n}$ and $U^{H}$ are the DOFs of the high-order finite element solution $u_{h}(\mathbf{x}, t)$ at time $t=t_{n}$ and $t=t_{n+1}$, respectively. 


\section{Low-Order MPP Method}

We consider the first-order MPP approach in $[18,19]$. This method is based on applying a discrete upwinding to the transport matrix $K$ of a high-order scheme and lumping the mass matrix $M$. This leads to

$$
M^{*}\left(\frac{U^{L}-U^{n}}{\Delta t}\right)=K^{*} U^{n}
$$

where $M^{*}$ and $K^{*}$ are the lumped mass matrix and the upwinded transport matrix respectively. They are given as follows:

$$
\begin{aligned}
M^{*} & =M+L, \\
K^{*} & =K+D,
\end{aligned}
$$

where $L$ and $D$ are given by

$$
L_{i j}=-M_{i j}, \quad D_{i j}=\max \left(0,-K_{i j},-K_{j i}\right),
$$

for the off-diagonal elements and

$$
L_{i i}=-\sum_{j \neq i} L_{i j}, \quad D_{i i}=-\sum_{j \neq i} D_{i j}
$$

otherwise. Note that the lumping and upwinding are algebraic processes, and therefore the corresponding low-order solution depends on the choice of the basis functions. The matrices $L$ and $-D$ are algebraic diffusion matrices, i.e., they are symmetric, have non-positive off-diagonal entries and have zero row and column sum. These are the typical characteristics of the discretization of the Laplace operator $-\Delta[18]$. A critical property of our DG method is that the matrices $L$ and $D$ are block diagonal with $L_{i j}=D_{i j}=0$ if nodes $i$ and $j$ belong to different mesh cells (see Section 6.1). The size of diagonal blocks is given by the number of DOFs per element. The method is first order accurate. Mass conservation in (7) follows from the fact that both $K$ and $D$ have zero column sums. In praticular, $M^{*}$ and $M$ have the same rowsums, so the mass is conserved with respect to the original high-order mass matrix.

One can see that the above method is MPP by rewriting (7) as

$$
U_{i}^{L}=\sum_{j} R_{i j} U_{j}^{n}
$$

Here the off-diagonal entries of $R_{i j}=\left[\left(M^{*}\right)^{-1}\left(M^{*}+\Delta t K^{*}\right)\right]_{i j}$ are positive by the construction of $M^{*}$ and $K^{*}$, and the diagonal ones can be made positive by choosing $\Delta t$ small enough. In addition, if $\mathbb{1}$ is the vector of ones, then

$$
R \mathbb{1}=\left(M^{*}\right)^{-1}\left(M^{*}+\Delta t K^{*}\right) \mathbb{1}=\left(\mathbb{1}+\Delta t\left(M^{*}\right)^{-1} K^{*} \mathbb{1}\right)=\mathbb{1},
$$

which is true since $K^{*} \mathbb{1}=(K+D) \mathbb{1}=0$. Therefore, for any $i=1, \ldots, N, U_{i}^{L}$ is a convex combination of $U^{n}$. 


\subsection{Low-order MPP method with positive v.s. non-positive basis functions}

In this subsection we motivate our choice of the positive Bernstein polynomials, see Section 2.2 in [4], as finite element basis functions. Additional arguments are given in Section 6.1, which shows that the use of Bernstein polynomials results in high-order flux corrections that conserve the mass cell-wise, a crucial characteristic we exploit in this scheme.

Given a solution with coefficients $\left\{U_{1}, \ldots, U_{N}\right\}$, one is often interested in evaluating the solution at a set of quadrature points (which may not be known a priori), for example, when coupling advection with other PDEs. It is generally desirable for these solution values to be in bounds, i.e. inside the interval $\left[\min _{i} U_{i}, \max _{i} U_{i}\right]$. Consider a finite dimensional space $X_{h}$ and let $\left\{\phi_{1}, \ldots, \phi_{N}\right\}$ be a basis of $X_{h}$. The solution $u_{h} \in X_{h}$, at a point $\mathbf{x} \in \Omega$, is obtained via the linear combination $u_{h}(\mathbf{x})=\sum_{i} U_{i} \phi_{i}(\mathbf{x})$. It is clear that the solution $u_{h}(\mathbf{x})$ is guaranteed to be in bounds provided that $\sum_{i} U_{i} \phi_{i}(\mathbf{x})$ is a convex combination of $\left\{U_{1}, \ldots, U_{N}\right\}$; i.e., the shape functions $\left\{\phi_{1}, \ldots, \phi_{N}\right\}$ are positive $\forall \mathbf{x} \in \Omega$ and form a partition of unity. Both of these requirements are satisfied by the Bernstein shape functions.

Remark 3.1.1. Note that the Bernstein basis is generally not interpolatory, i.e., $U_{i}$ is not necessarily equal to the value of $u_{h}$ at some point $\mathbf{x}_{i}$. Only the coefficients $U_{i}$ associated with the vertices of the mesh are interpolatory. Also, the fact that the basis has local support, guarantees the cell-localization of the bounds: the value $u_{h}(\mathbf{x})$ is bounded below and above by the minimum and maximum of the coefficients associated only with the mesh cell that contains the point $\mathbf{x}$.

Furthermore, our numerical experiments indicate that within the method described by equations (7), positive basis functions give low-order solutions of better quality. To demonstrate this, we consider $\Omega=(0,1) \subset \mathbb{R}$, with velocity $\mathbf{v}=1$ and initial condition given by $u(\mathbf{x}, t=0)=\cos (2 \pi(x-0.5))$. Periodic boundary conditions are imposed, and the initial condition is used as exact solution at $T=1$. Figure 2 compares the Bernstein solutions to the ones obtained by the Gauss-Legendre and GaussLobatto nodal bases. The solution is qualitatively similar for lower order polynomials. However, as we increase the order, the quality of the solution with nodal basis functions is highly reduced. With Gauss-Legendre the solution is extremely dissipated for the larger order polynomials. With GaussLobatto the solution is also more dissipated than if positive basis functions are used but not as much as with Gauss-Legendre; however, the solution is less smooth than before.

It is also important to note that even with positive basis functions the solution is more dissipated as we increase the order of the polynomials. To further study this we consider the same experiment with the same polynomial spaces but increase the number of DOFs. In Figure 3 we show the solution with 768, 1536 and 3072 DOFs. In all cases we obtained more dissipated solutions as the order of the polynomial space is increased. However, the solutions get closer as we increase the order. Convergence study for the Bernstein basis is presented in Table 1. One can see in this table that the convergence rate is slightly increased as the order is increased. From these two results we expect that using higher-order polynomials eventually gives better results. However, the resolution needed might be too large. This is important to consider when the low-order method is used within the FCT methodology; i.e., this behavior influences the quality of the high-order solution as we increase the order of the polynomial space. 


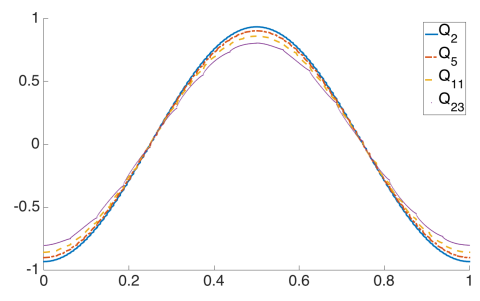

(a) Positive basis functions

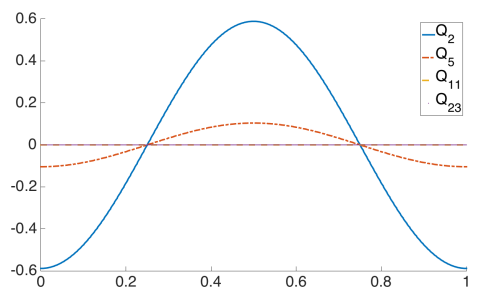

(b) Gauss-Legendre nodal basis

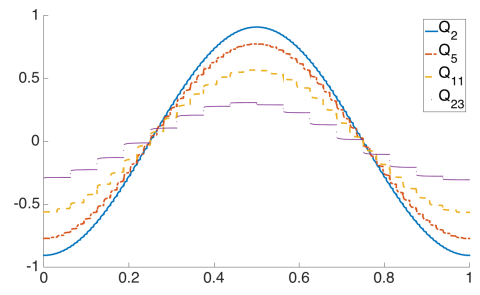

(c) Gauss-Lobatto nodal basis

Figure 2: 1D transport of a smooth function via the low-order method (7) using positive v.s. nodal basis functions. In each case we consider polynomial spaces $\mathbb{Q}_{2}, \mathbb{Q}_{5}, \mathbb{Q}_{11}$ and $\mathbb{Q}_{23}$. The number of cells is adjusted to have 384 DOFs in all simulations.

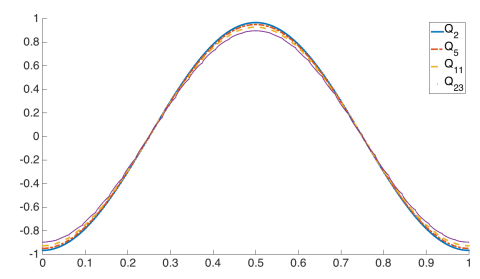

(a) 768 DOFs

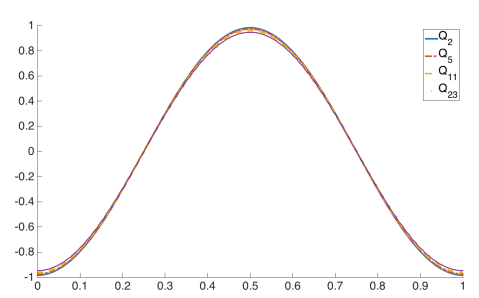

(b) 1536 DOFs

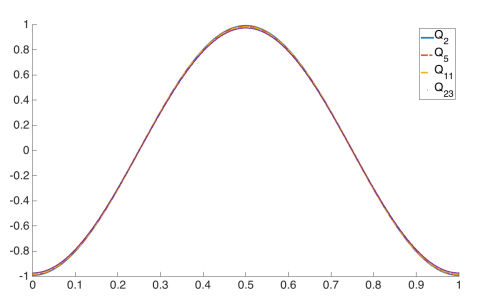

(c) 3072 DOFs

Figure 3: 1D transport of a smooth function via the low-order method (7) using positive basis functions with polynomial spaces $\mathbb{Q}_{2}, \mathbb{Q}_{5}, \mathbb{Q}_{11}$ and $\mathbb{Q}_{23}$. The number of cells is adjusted to have (a) 768, (b) 1536 and (c) 3072 DOFs.

\begin{tabular}{|c|c|c|c|c|c|c|}
\hline Cells & $\mathbb{Q}_{1}$ & conv & $\mathbb{Q}_{2}$ & conv & $\mathbb{Q}_{3}$ & conv \\
\hline 32 & $1.708 \mathrm{E}-01$ & & $1.534 \mathrm{E}-01$ & & $1.385 \mathrm{E}-01$ & \\
\hline 64 & $9.163 \mathrm{E}-02$ & 0.898 & $8.186 \mathrm{E}-02$ & 0.906 & $7.340 \mathrm{E}-02$ & 0.916 \\
\hline 128 & $4.752 \mathrm{E}-02$ & 0.947 & $4.231 \mathrm{E}-02$ & 0.952 & $3.780 \mathrm{E}-02$ & 0.957 \\
\hline 256 & $2.420 \mathrm{E}-02$ & 0.974 & $2.151 \mathrm{E}-02$ & 0.976 & $1.918 \mathrm{E}-02$ & 0.979 \\
\hline \hline Cells & $\mathbb{Q}_{5}$ & conv & $\mathbb{Q}_{11}$ & conv & $\mathbb{Q}_{23}$ & conv \\
\hline 32 & $1.189 \mathrm{E}-01$ & & $8.942 \mathrm{E}-02$ & & $6.585 \mathrm{E}-02$ & \\
\hline 64 & $6.247 \mathrm{E}-02$ & 0.928 & $4.641 \mathrm{E}-02$ & 0.946 & $3.383 \mathrm{E}-02$ & 0.961 \\
\hline 128 & $3.202 \mathrm{E}-02$ & 0.964 & $2.364 \mathrm{E}-02$ & 0.973 & $1.715 \mathrm{E}-02$ & 0.981 \\
\hline 256 & $1.622 \mathrm{E}-02$ & 0.982 & $1.193 \mathrm{E}-02$ & 0.987 & $8.630 \mathrm{E}-03$ & 0.990 \\
\hline
\end{tabular}

Table 1: $L^{1}(\Omega)$-convergence of low-order method (7). 


\section{Edge-Based Flux Corrected Transport (DG-FCT)}

In this section we revisit the Flux Corrected Transport methodology by [6] and [27], we also refer to [16] for more details. This method interpolates between a low-order MPP and a high-order nonMPP solution. The resulting method is denoted as DG-FCT throughout this work.

The high-order method (6) can be rewritten as

$$
m_{i}\left(U_{i}^{H}-U_{i}^{L}\right)=\sum_{j}\left(M^{*}-M\right)_{i j}\left(U_{j}^{H}-U_{j}^{n}\right)-\Delta t D_{i j} U_{j}^{n}
$$

where $U_{i}^{L}$ is the low-order solution given by (7), and the right hand side is a flux correction. Note that for any $i=1, \ldots, N, \sum_{j}\left(M^{*}-M\right)_{i j}=0$ and $\sum_{j} D_{i j}=0$, by the properties of the lumped matrix $M^{*}$ and the diffusive operator $D$. Therefore,

$$
\sum_{j} D_{i j} U_{j}^{n}=\sum_{j \neq i} D_{i j} U_{j}^{n}+D_{i i} U_{i}^{n}=\sum_{j \neq i}\left(U_{j}^{n}-U_{i}^{n}\right) D_{i j}=\sum_{j}\left(U_{j}^{n}-U_{i}^{n}\right) D_{i j} .
$$

Since $D$ is symmetric, the pairs $\left[\left(U_{j}^{n}-U_{i}^{n}\right) D_{i j},\left(U_{i}^{n}-U_{j}^{n}\right) D_{j i}\right]$ form an anti-symmetric matrix. Similarly,

$$
\sum_{j}\left(M^{*}-M\right)_{i j}\left(U_{j}^{H}-U_{j}^{n}\right)=\sum_{j}\left(M^{*}-M\right)_{i j}\left(\delta U_{j}-\delta U_{i}\right)
$$

where $\delta U:=U^{H}-U^{n}$. Here the pairs $\left[\left(M^{*}-M\right)_{i j}\left(\delta U_{j}-\delta U_{i}\right),\left(M^{*}-M\right)_{j i}\left(\delta U_{i}-\delta U_{j}\right)\right]$ also form an anti-symmetric matrix. Let

$$
f_{i j}:=\left(M^{*}-M\right)_{i j}\left(\delta U_{j}-\delta U_{i}\right)-\Delta t D_{i j}\left(U_{j}^{n}-U_{i}^{n}\right) .
$$

Then the high-order method (6) can be written as

$$
U_{i}^{H}=U_{i}^{L}+m_{i}^{-1} \sum_{j \neq i} f_{i j}
$$

In this form, it is clear that flux correction improves the accuracy of the low-order method to make it high-order. In addition, it is responsible for the high-order solution to be in bounds. The idea behind DG-FCT is to limit this correction whenever the solution bounds are violated. Doing this we get

$$
U_{i}^{n+1}=U_{i}^{L}+m_{i}^{-1} \sum_{j \neq i} \alpha_{i j} f_{i j}
$$

where $\alpha_{i j}$ 's are the flux limiters computed as follows:

$$
\alpha_{i j}:= \begin{cases}\min \left(R_{i}^{+}, R_{j}^{-}\right) & \text {if } f_{i j} \geq 0 \\ \min \left(R_{i}^{-}, R_{j}^{+}\right) & \text {otherwise }\end{cases}
$$


where

$$
\begin{aligned}
& R_{i}^{+}:=\left\{\begin{array}{ll}
\min \left(1, \frac{Q_{i}^{+}}{P_{i}^{+}}\right), & P_{i}^{+} \neq 0, \\
1 & \text { otherwise, }
\end{array} \quad R_{i}^{-}:= \begin{cases}\min \left(1, \frac{Q_{i}^{-}}{P_{i}^{-}}\right), & P_{i}^{-} \neq 0, \\
1 & \text { otherwise },\end{cases} \right. \\
& P_{i}^{+}:=\sum_{j} \max \left(0, f_{i j}\right), \quad P_{i}^{-}:=\sum_{j} \min \left(0, f_{i j}\right), \\
& Q_{i}^{+}:=m_{i}\left(U_{i}^{\max }-U_{i}^{L}\right), \quad Q_{i}^{-}:=m_{i}\left(U_{i}^{\min }-U_{i}^{L}\right) .
\end{aligned}
$$

Here $U_{i}^{\max }$ and $U_{i}^{\min }$ are defined as local maxima and minima of $U^{L}$ (see below).

We refer to [4] for a numerical validation of the DG-FCT method using the low- and the high-order methods (7) and (6), respectively.

Mass conservation follows from the symmetry properties of $\alpha_{i j}$ and $f_{i j}$, namely, the row sum of (11) is

$$
\sum_{i} m_{i}\left(U_{i}^{n+1}-U_{i}^{L}\right)=\sum_{i} \sum_{j \neq i} \alpha_{i j} f_{i j}=\sum_{i, j>i} \alpha_{i j} f_{i j}+\alpha_{j i} f_{j i}=\sum_{i, j>i} \alpha_{i j}\left(f_{i j}-f_{i j}\right)=0 .
$$

Remark 4.0.2 (Maximum Principle Preservation). Assume that $U^{L}$ satisfies the local discrete maximum principle; i.e., $U_{i}^{\min } \leq U_{i}^{L} \leq U_{i}^{\max }$ for all $i=1, \ldots, N$. Then the solution of (11) satisfies the local discrete maximum principle; i.e., $U_{i}^{\min } \leq U_{i}^{n+1} \leq U_{i}^{\max }$ for all $i=1, \ldots, N$. To see this we follow $[9,18]$. Assume that $P_{i}^{+} \neq 0$, then using (12) we get

$$
\begin{aligned}
m_{i}\left(U_{i}^{n+1}-U_{i}^{L}\right) & =\sum_{j} \alpha_{i j} f_{i j} \leq \sum_{j \neq i, f_{i j} \geq 0} \alpha_{i j} f_{i j}=\sum_{j \neq i, f_{i j} \geq 0} \min \left(R_{i}^{+}, R_{j}^{-}\right) f_{i j} \leq \sum_{j \neq i, f_{i j} \geq 0} R_{i}^{+} f_{i j} \\
& \leq \frac{Q_{i}^{+}}{P_{i}^{+}} \sum_{j \neq i, f_{i j} \geq 0} f_{i j}=\frac{Q_{i}^{+}}{P_{i}^{+}} \sum_{j \neq i} \max \left(0, f_{i j}\right)=Q_{i}^{+}=m_{i}\left(U_{i}^{\max }-U_{i}^{L}\right) ;
\end{aligned}
$$

therefore, $U_{i}^{n+1} \leq U_{i}^{\max }$. If $P_{i}^{+}=0$, then

$$
m_{i}\left(U_{i}^{n+1}-U_{i}^{L}\right) \leq \sum_{j \neq i, f_{i j} \geq 0} R_{i}^{+} f_{i j}=R_{i}^{+} \sum_{j \neq i} \max \left(0, f_{i j}\right)=R_{i}^{+} P_{i}^{+}=0
$$

for any $R_{i}^{+}$. Provided $U_{i}^{L} \leq U_{i}^{\max }$, we get $P_{i}^{+}=0 \leq m_{i}\left(U_{i}^{\max }-U_{i}^{L}\right)$, which implies $U_{i}^{n+1} \leq U_{i}^{\max }$. The lower bound $U_{i}^{\min } \leq U_{i}^{n+1}$ is proven similarly.

The DG-FCT methodology, as revisited here, produces good quality results and recovers the full accuracy of the high-order methods when the polynomial spaces are of relatively low-order. However, as the order of the polynomials is increased spurious oscillations are introduced. This is true even though the method is still MPP. To illustrate this problem we consider a 1D problem with non-smooth initial data

$$
u_{h}(x, t=0)= \begin{cases}1, & \forall x \in(0.4,0.6) \\ 0, & \text { otherwise }\end{cases}
$$


over $\Omega=(0,1) \subset \mathbb{R}$ and velocity given by $v=1$ and obtain the solution using different polynomial spaces. The results are shown in Figure 4.

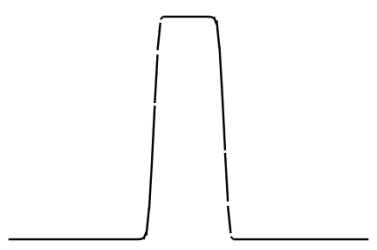

$\mathbb{Q}_{1}$ with 256 DOFs

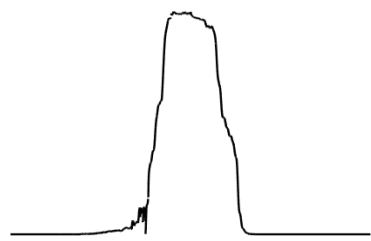

$\mathbb{Q}_{5}$ with 768 DOFs

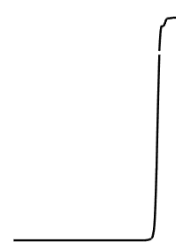

$\mathbb{Q}_{2}$ with 384 DOFs

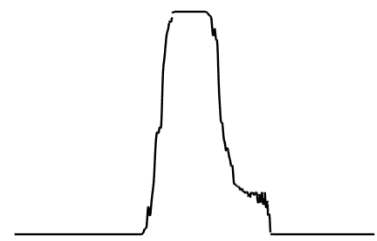

Q6 with 896 DOFs

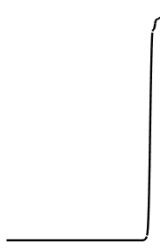

$\mathbb{Q}_{3}$ with 512 DOFs

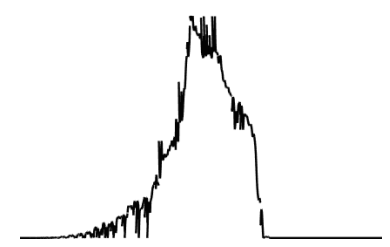

$\mathbb{Q}_{7}$ with 1024 DOFs

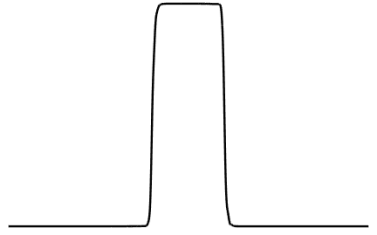

$\mathbb{Q}_{4}$ with 640 DOFs

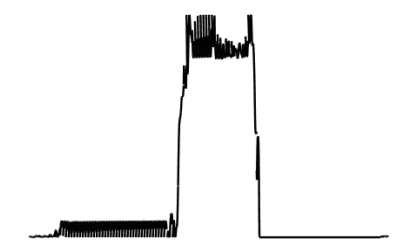

$\mathbb{Q}_{8}$ with 1152 DOFs

Figure 4: 1D simulations with non-smooth initial data using the DG-FCT method (11) for different polynomial spaces. In all simulations 128 cells are used and, therefore, they have different number of DOFs.

In addition, we consider a more complicated non-smooth initial condition in 2D, shown in Figure 5, with $\Omega=(0,100) \times(0,100) \subset \mathbb{R}^{2}$ and velocity $\mathbf{v}=(10,10)$. We compute the solution at time $t=4$ using $\mathbb{Q}_{2}$ and $\mathbb{Q}_{5}$ spaces with the cells adjusted to have the same number of DOFs. The results are shown in Figure 5.

The problem is clear, as we consider higher-order spaces the oscillations become more drastic making the solution unacceptable. In the remainder of this work we propose various approaches to reduce these oscillations.

\subsection{DG-FCT Algorithm Summary}

Now we summarize the major DG-FCT steps performed inside a single Runge-Kutta stage. Specific implementations can be derived that combine or interchange them in order to improve performance and reuse data structures.

1. Assembly of the sparse matrices $M$ and $K$, as well as computation of $m_{i}=M_{i i}^{*}$ and $K_{i i}^{*}$ for every index $i$.

2. Computation of $\Delta t$, so that $m_{i}+\Delta t K_{i i}^{*} \geq 0$ for every index $i$.

3. Given $U^{n}$ and the sparsity of $K,(2)$ is used to compute $U^{\min }$ and $U^{\max }$.

4. Computation of $U^{L}$ by (7), $U^{H}$ by (6) and the fluxes $f_{i j}$ by (9). 


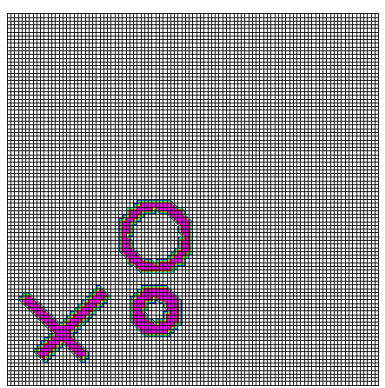

(a) $\mathbb{Q}_{2}, \quad(\min , \max )=\left(-2.587 \times 10^{-15}, 1.99\right)$

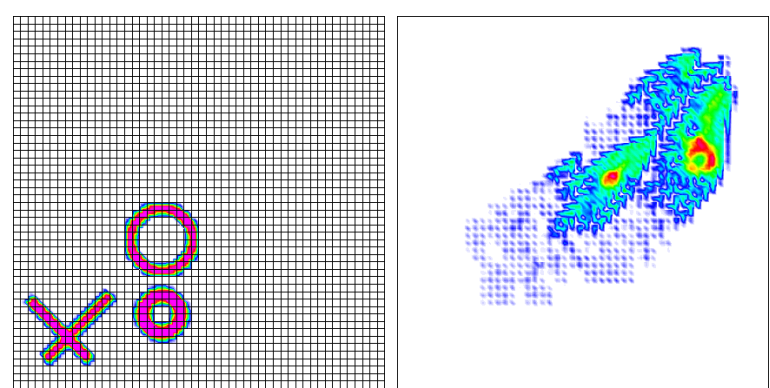

(b) $\mathbb{Q}_{5}, \quad(\min , \max )=\left(-1.363 \times 10^{-14}, 1.632\right)$

Figure 5: 2D simulations with non-smooth initial data using the DG-FCT method (11) for $\mathbb{Q}_{2}$ and $\mathbb{Q}_{5}$ spaces. The number of cells is adjusted so that 90000 DOFs are used in both simulations. For each case, we show (left) the initial condition with the grid and (right) the solution at $t=4$.

5. Computation of $R^{+}$and $R^{-}$by (12b), and their substitution in (11) to obtain $U^{n+1}$.

Note that the first two steps should be performed only once, since they are independent of time.

\section{Localized DG-FCT}

The DG-FCT method from Section 4 is local in the sense given by the sparsity pattern of the transport matrix $K$. For Discontinuous Galerkin finite elements this sparsity pattern includes all DOFs on a given cell and on cells sharing a face with it, see Figure 6a. When the order of the polynomial space is small, the sparsity pattern includes few DOFs; however, as we increase the order, the number of DOFs in the sparsity pattern increases. The method loses locality with respect to DOFs; nevertheless, it is fixed with respect to number of cells. In an extreme case we can consider a single cell with a polynomial space of order as large as needed to have roughly certain number of DOFs; in this situation, the local and global maximum principles are equivalent, since the sparsity pattern includes all DOFs in the finite element space. This motivates the idea of considering tighter bounds. We propose to localize the bounds by mimicking the stencil of a first-order space; i.e., for a given $i$-th DOF we consider those at locations adjacent to $i$. Let $N_{i}$ be the conventional neighborhood for the $i$-th DOF. For the finite element space $\mathbb{Q}_{k}$, we use tighter bounds given by the stencil

$$
N_{i}^{*}=\left\{j \in N_{i}: \operatorname{dist}(i, j) \leq \frac{1}{k} \sqrt{d}\right\}
$$

where $d \in\{1,2,3\}$ is the space dimension and $\operatorname{dist}(i, j)$ is the Euclidean distance between the two DOFs' images on the reference element. Defining $N_{i}^{*}$ with respect to the reference element makes the approach applicable to unstructured grids. In Figure 6, we consider a representative DOF in thick blue and show the conventional or full stencil via the sparsity pattern of the transport matrix $K$; in 
addition, we show the tighter bounds (14), mimicking a stencil for a first-order space. Note that since DG discretization is used we have two DOFs at the faces, this is denoted by using a red circle and a black cross in those locations.

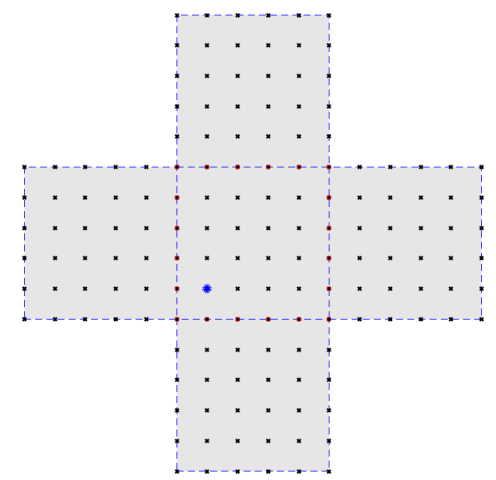

(a) Full stencil

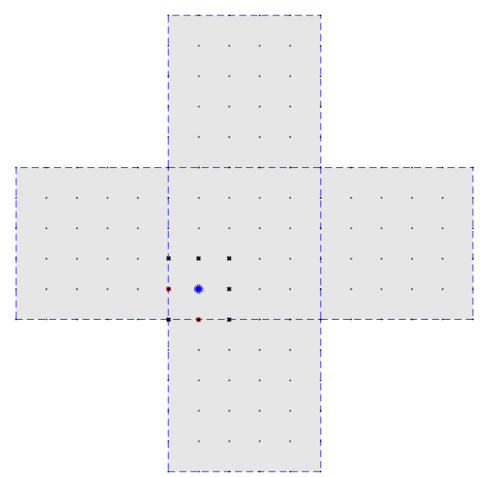

(b) First-order stencil

Figure 6: Stencil to compute bounds for a representative DOF. In (a) we show the conventional or full stencil in a DG discretization. In (b) we mimic a $\mathbb{Q}_{1}$ space. The thick blue mark represents the DOF for which we compute the bounds, the thick black marks represent the DOFs included to compute the bounds, the non-thick black dots in (b) represent all DOFs in the sparsity pattern of $K$ and the red marks indicate a double DOF for the given location (they are also considered for the computation of the bounds).

Remark 5.0.1 (Low-order method is non-MPP in the tighter bounds). The low-order method (7) is guaranteed to produce an MPP solution in the conventional bounds; i.e., including all DOFs in the sparsity pattern of $K$. Since the tighter bounds considers a smaller set of DOFs there is no guarantee the low-order solution is MPP in this set.

Due to remark 5.0.1, we can't use the DG-FCT methodology with the bounds in (2) given by the tighter stencil (14). To overcome this we modify the bounds in (2) to be

$$
\begin{aligned}
& U_{i}^{\min }=\min \left(U_{i}^{L}, \min _{j \in N_{i}^{*}} U_{j}^{n}\right), \\
& U_{i}^{\max }=\max \left(U_{i}^{L}, \max _{j \in N_{i}^{*}} U_{j}^{n}\right),
\end{aligned}
$$

which guarantees the low-order solution is in bounds and, therefore, we can apply the DG-FCT methodology. We now repeat the simulations from Section 4 using the tighter bounds (15). The results are shown in Figures 7 and 8. We observe the oscillatory behavior, although not completely eliminated, is highly reduced. It is clear also the high amount of dissipation introduced as the order of the polynomial space is increased. In the next section we propose an FCT-like methodology that reduces even more the oscillatory behavior and yields less dissipated solutions. 
Remark 5.0.2. Localized bounds are used to provide better control over function values $u_{h}(\mathbf{x})$, but satisfying the localized bounds for $U_{i}$ does not guarantee that $u_{h}\left(\mathbf{x}_{i}\right)$ will be within these bounds, see Remark 3.1.1. This is because

$$
u_{h}\left(\mathbf{x}_{i}\right)=\sum_{j \in N_{K_{i}}} U_{j} \phi\left(\mathbf{x}_{i}\right)
$$

is formed over a wider stencil than the one used to define its bounds. Here $K_{i}$ is the cell containing $\mathbf{x}_{i}$ and $N_{K_{i}}=\left\{j: \mathbf{x}_{j} \in K_{i}\right\}$ is the set of DOF indices associated with $K_{i}$.

Nevertheless, the additional control is sufficient to improve significantly the behavior of high-order polynomials, as one can observe in Figures 7 and 8.

Remark 5.0.3 (Triangular and tetrahedral grids). Although the tests in this paper are focused on quadrilateral and hexahedral grids, all presented finite element methods are also applicable to triangular and tetrahedral meshes. In this case, the localized bounds can be defined by

$$
N_{i}^{*}=\left\{j \in N_{i}: \operatorname{dist}(i, j) \leq \frac{1}{k}\right\}
$$

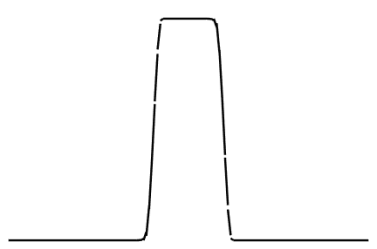

$\mathbb{Q}_{1}$ with 256 DOFs

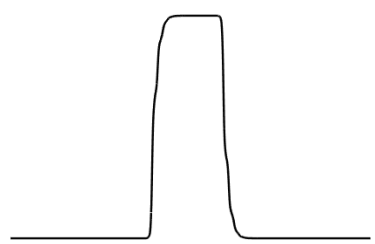

$\mathbb{Q}_{5}$ with 768 DOFs

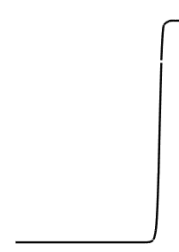

$\mathbb{Q}_{2}$ with 384 DOFs

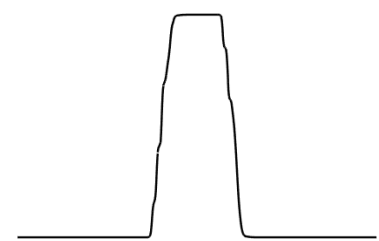

$\mathbb{Q}_{6}$ with 896 DOFs

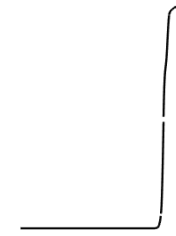

$\mathbb{Q}_{3}$ with 512 DOFs

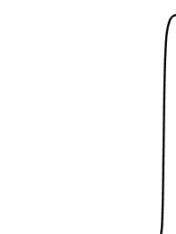

$\mathbb{Q}_{7}$ with 1024 DOFs

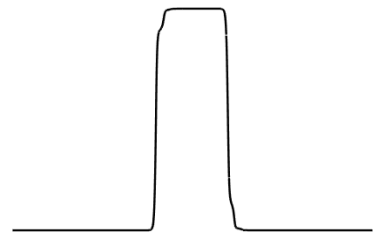

$\mathbb{Q}_{4}$ with 640 DOFs

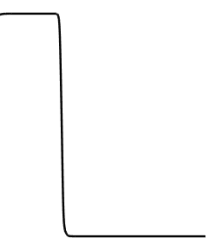

$\mathbb{Q}_{8}$ with 1152 DOFs

Figure 7: 1D simulations with non-smooth initial data using the localized DG-FCT with bounds (15). The low- and high-order methods are given by (7) and (6), respectively. We consider different polynomial spaces. In all simulations 128 cells are used and, therefore, they have different number of DOFs.

\section{Element-Based Flux Corrected Transport (DG-EFCT)}

In the DG-FCT method revisited in Section 4, we start with two methods that are mass conservative. One is low-order and MPP, and the other is high-order, but non-MPP. Then, an interpolation is made 


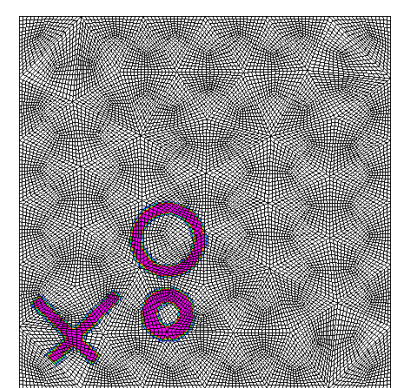

(a) $\mathbb{Q}_{2}, \quad(\min , \max )=\left(-2.309 \times 10^{-17}, 1.976\right)$

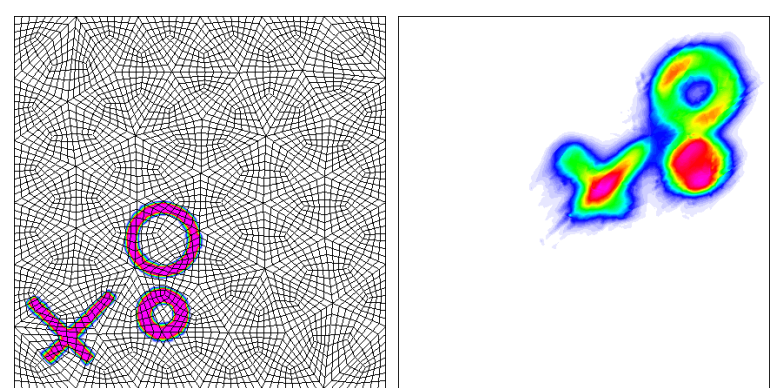

(b) $\mathbb{Q}_{5}, \quad(\min , \max )=\left(-1.686 \times 10^{-20}, 1.322\right)$

Figure 8: 2D simulations with non-smooth initial data using the localized DG-FCT method with bounds (15) on an unstructured grid. The low- and high-order methods are given by (7) and (6), respectively. We use $\mathbb{Q}_{2}$ and $\mathbb{Q}_{5}$ spaces with the number of cells adjusted so that 127872 DOFs are used in both simulations. For each case, we show (left) the initial condition with the grid and (right) the solution at $t=4$.

from the low- to the high-order solution to obtain a solution that is MPP. For any DOF $U_{i}^{n+1}$, there are as many interpolating parameters as DOFs in the neighborhood of $U_{i}^{n+1}$. These interpolating parameters are designed in a way that conserves mass.

In this work we present an FCT-like method (which we denote by DG-EFCT) that considers two MPP solutions. One is low-order and mass conservative, and the other is (presumably) high-order, but non-conservative. Then, we interpolate from the low- to the high-order solution to recover mass conservation cell-wise. In contrast to DG-FCT, in this method, for any DOF $U_{i}^{n+1}$ we have just one interpolating parameter. This interpolating parameter is designed to maintain the solution in bounds. It is important to emphasize that the recovery in mass conservation is obtained per cell and not globally. Moreover, we propose a methodology to localize even more this redistribution of mass inside a cell. Recovering the conservation of mass within a cell is possible due to local mass properties of the low- and high-order methods we consider in this work and by using Discontinuous Galerkin spatial discretization. We explain this in more detail in the next section.

\subsection{Mass conservation of low- and high-order methods}

In this section, we show that the low- and the high-order solutions in (7) and (6) have the same mass on any given cell $K \in \mathcal{T}_{h}$; i.e., $\sum_{i \in N_{K}} m_{i} U_{i}^{H}=\sum_{i \in N_{K}} m_{i} U_{i}^{L}$, where $N_{K}=\left\{i: \mathbf{x}_{i} \in K\right\}$ is the set of node numbers for the DOFs associated with $K$. To prove this, we rely on the specific DG finite element formulation as well as the use of the positive shape functions described in Section 2. First, we rewrite the high-order method as

$$
m_{i}\left(U_{i}^{H}-U_{i}^{L}\right)=f_{i}^{H},
$$


where $f_{i}^{H}$ is a high-order flux correction given by

$$
f_{i}^{H}=\sum_{j \in N_{i}}\left(M^{*}-M\right)_{i j} \delta U_{j}-\Delta t \sum_{j \in N_{i}} D_{i j} U_{j}^{n}
$$

Here $\delta U_{j}:=U_{j}^{H}-U_{j}^{n}$. Given any cell $K \in \mathcal{T}_{h}$ consider

$$
\sum_{i \in N_{K}} f_{i}^{H}=\sum_{j \in N_{i}} \delta U_{j} \sum_{i \in N_{K}}\left(M^{*}-M\right)_{i j}-\Delta t \sum_{j \in N_{i}} U_{j} \sum_{i \in N_{K}} D_{i j} .
$$

It is our aim to show that $\sum_{i \in N_{K}} f_{i}^{H}=0 \Longrightarrow \sum_{i \in N_{K}} m_{i} U_{i}^{H}=\sum_{i \in N_{K}} m_{i} U_{i}^{L}$. Since we use a DG discretization, all shape functions are supported on a single cell. Therefore, $M_{j j}^{*}=\sum_{i=1, \ldots, N} M_{i j}=$ $\sum_{i \in N_{K}} M_{i j}$ and, hence, $\sum_{i \in N_{K}}\left(M^{*}-M\right)_{i j}=0$.

From Section 3, we recall that $\sum_{i \in[1, \ldots, N]} D_{i j}=0$. Now we show that $D_{i j}=0$ whenever $i$ and $j$ belong to different cells. Recall the definition of $D_{i j}$ from Section 3:

$$
D_{i j}=\max \left(0,-K_{i j},-K_{j i}\right) \text { if } i \neq j, \quad D_{i i}=-\sum_{j \neq i} D_{i j}
$$

We just need to consider the off-diagonal elements and assume they belong to different cells. From Section 2.1, the $i, j$-th element of the transport matrix is given by

$$
K_{i j}=-\int_{\Omega} \phi_{j}\left(\mathbf{v} \cdot \nabla \phi_{i}\right) d \mathbf{x}+\sum_{f \in \mathcal{F}_{h}} \int_{f}\left\{\phi_{j} \mathbf{v} \cdot \mathbf{n}_{f}\right\}_{*}\left[\left[\phi_{i}\right]\right] d \mathbf{s},
$$

where the first integral is zero since $i$ and $j$ belong to different cells and each shape function is supported on its corresponding cell. Recall the definition of the numerical flux:

$$
\begin{aligned}
\left\{\phi \mathbf{v} \cdot \mathbf{n}_{F}\right\}_{*} & =\left(\mathbf{v} \cdot \mathbf{n}_{F}\right)\left(\frac{\left.\phi\right|_{K_{1}}+\left.\phi\right|_{K_{2}}}{2}\right)-\frac{1}{2}\left|\mathbf{v} \cdot \mathbf{n}_{F}\right|[[\phi]], \\
{[[\phi]] } & =\phi^{-}-\phi^{+} \\
\phi^{ \pm}(\mathbf{x}) & =\lim _{\xi \rightarrow 0^{+}} \phi\left(\mathbf{x} \pm \xi \mathbf{n}_{f}(\mathbf{x})\right) .
\end{aligned}
$$

Suppose the normal vector $\mathbf{n}_{F}$ points from cell $K_{1}$ to cell $K_{2}$. Then we get $[[\phi]]=\left.\phi\right|_{K_{1}}-\left.\phi\right|_{K_{2}}$. Assume $\phi_{j}$ is supported on cell $K_{1}$ and $\phi_{i}$ on cell $K_{2}$, then $\left[\left[\phi_{j}\right]\right]=\left.\phi_{j}\right|_{K_{1}}$ and $\left[\left[\phi_{i}\right]\right]=-\left.\phi_{i}\right|_{K_{2}}$, which leads to

$$
\left\{\phi_{j} \mathbf{v} \cdot \mathbf{n}_{F}\right\}_{*}\left[\left[\phi_{i}\right]\right]=\left[\left|\mathbf{v} \cdot \mathbf{n}_{F}\right|-\left(\mathbf{v} \cdot \mathbf{n}_{F}\right)\right]\left(\frac{\left.\left.\phi_{j}\right|_{K_{1}} \phi_{i}\right|_{K_{2}}}{2}\right),
$$

which is non-negative regardless of the sign of $\mathbf{v} \cdot \mathbf{n}_{F}$ provided the shape functions are positive. Similarly, if $\phi_{j}$ is supported on cell $K_{2}$ and $\phi_{i}$ on cell $K_{1}$ we get

$$
\left\{\phi_{j} \mathbf{v} \cdot \mathbf{n}_{F}\right\}_{*}\left[\left[\phi_{i}\right]\right]=\left[\left(\mathbf{v} \cdot \mathbf{n}_{F}\right)+\left|\mathbf{v} \cdot \mathbf{n}_{F}\right|\right]\left(\frac{\left.\left.\phi_{j}\right|_{K_{2}} \phi_{i}\right|_{K_{1}}}{2}\right),
$$


which is also non-negative provided we use positive shape functions. Therefore, $K_{i j} \geq 0$ whenever $i$ and $j$ don't belong to the same cell. This implies that $D_{i j}=0$ whenever $i$ and $j$ don't belong to the same cell.

From $\sum_{i \in[1, \ldots, N]} D_{i j}=0$ and $D_{i j}=0$ whenever $i$ and $j$ don't belong to the same cell we conclude that $\sum_{i \in N_{K}} D_{i j}=0$. Therefore, we get

$$
\sum_{i \in N_{K}} f_{i}^{H}=\sum_{j} \delta U_{j} \sum_{i \in N_{K}}\left(M^{*}-M\right)_{i j}-\Delta t \sum_{j} U_{j} \sum_{i \in N_{K}} D_{i j}=0 .
$$

The property that the mass of the high-order flux correction is zero for any given cell is crucial for the method presented in this work. This allows us to consider non mass conservative flux corrections that assure the solution is in bounds and then adjust those fluxes to recover mass conservation per cell. To do this we need to adjust the fluxes on any cell without modifying fluxes in other cells. This is possible since we consider DG finite elements.

\subsection{Clipped solution}

The first stage of this method is to clip the solution considering some local bounds. We can consider different options depending on the stencil; i.e., we can consider the full or conventional stencil $N_{i}$ (figure $6 \mathrm{a}$ ) or the tighter stencil $N_{i}^{*}$ from equation (14) (figure 6b). In either case we obtain $\left\{U_{i}^{\min }, U_{i}^{\max }\right\}$. Then we consider the high-order solution $U^{H}$ from method (6) to get

$$
U_{i}^{*}=\min \left(U_{i}^{\max }, \max \left(U_{i}^{H}, U_{i}^{\min }\right)\right),
$$

where $U_{i}^{*}$ is the clipped solution. Note that, since $N_{i}^{*} \subset N_{i}$, the clipped solution $U_{i}^{*}$ is in bounds in both the tight and the full stencil, i.e.,

$$
\min _{j \in N_{i}} U_{j}^{n} \leq \min _{j \in N_{i}^{*}} U_{j}^{n} \leq U_{i}^{*} \leq \max _{j \in N_{i}^{*}} U_{j}^{n} \leq \max _{j \in N_{i}} U_{j}^{n} .
$$

In Figure 9 we revisit the non-smooth 1D problem (13) and show the results of clipping the solution with the bounds computed via the full and the tighter stencil. We use $\mathbb{Q}_{5}$ and $\mathbb{Q}_{11}$ spaces. It is clear that non-physical oscillations are present when the full stencil is considered. For this reason we always compute the bounds (2) using the tighter stencil $N_{i}^{*}(14)$. Next, in Figure 10 we show results of the same problem considering different spaces and refinement levels. Two observations can be made from this figure. First, phase errors appear due to not conserving mass. Mass conservation is addressed in the next section. Second, the clipped solution becomes more dissipated as one considers higher order spaces.

\subsection{Local recovery of mass conservation}

In this section, we consider the clipped solution $U_{i}^{*}$ and recover mass conservation locally in each cell. In Section 6.1 we saw that the high-order flux correction

$$
f_{i}^{H}=m_{i}\left(U_{i}^{H}-U_{i}^{L}\right)
$$




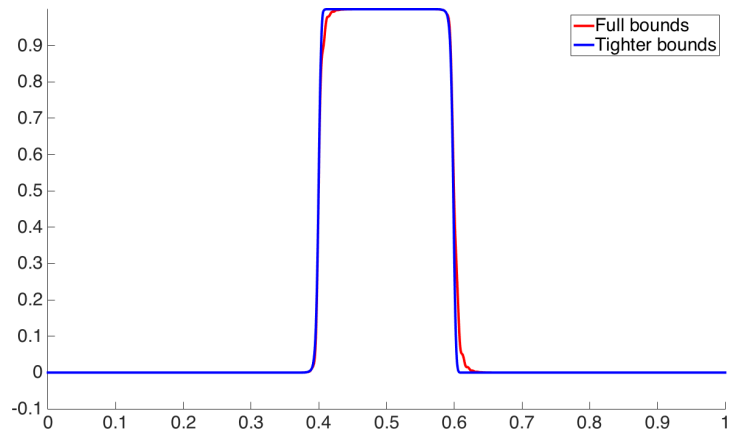

(a) $\mathbb{Q}_{5}$

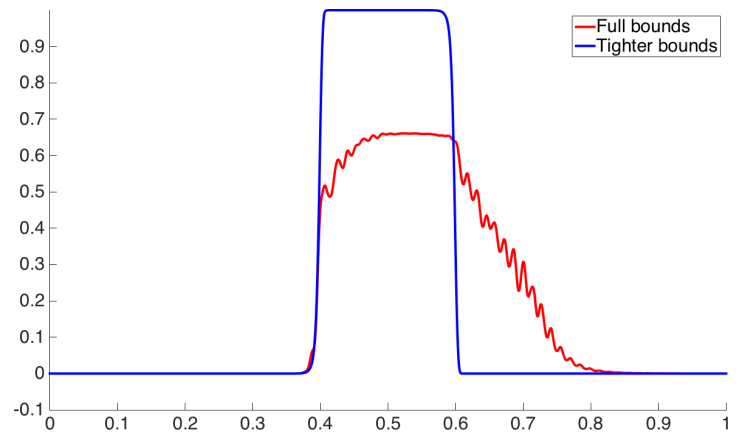

(b) $\mathbb{Q}_{11}$

Figure 9: Solution clipping via (20) for non-smooth initial data. We consider $\mathbb{Q}_{5}$ and $\mathbb{Q}_{11}$ spaces using the full and the tighter stencils. The number of cells is adjusted so that 768 DOFs are used in both simulations.

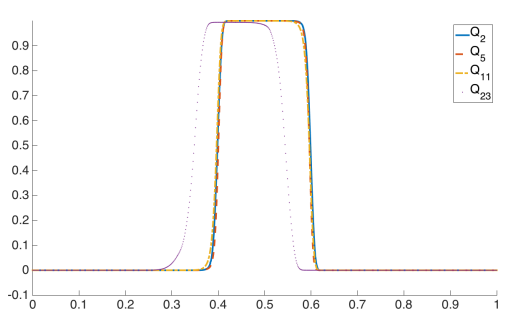

(a) 384 DOFs

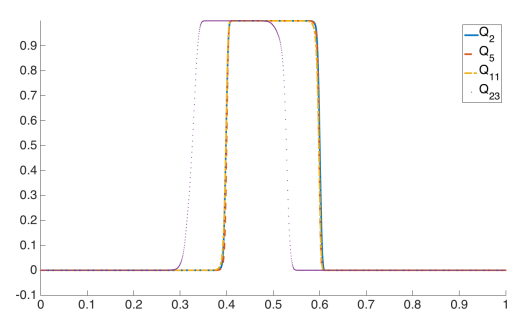

(b) 768 DOFs

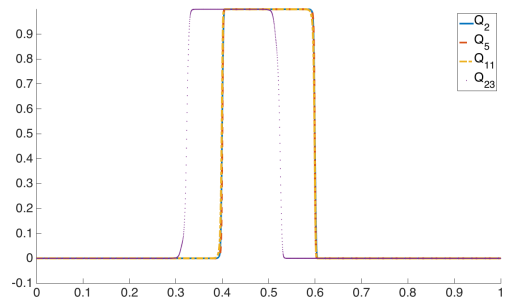

(c) 1536 DOFs

Figure 10: Solution clipping via (20) for non-smooth initial data. We consider $\mathbb{Q}_{2}, \mathbb{Q}_{5}, \mathbb{Q}_{11}$ and $\mathbb{Q}_{23}$ spaces using the tighter stencil. The number of cells is adjusted to have (a) 384, (b) 768 and (c) 1536 DOFs. 
has zero mass within a cell; i.e., $\sum_{i \in N_{K}} f_{i}^{H}=0, \forall K \in \mathcal{T}_{h}$. Given the clipped solution $U_{i}^{*}$, define

$$
f_{i}^{*}:=m_{i}\left(U_{i}^{*}-U_{i}^{L}\right)
$$

Here $f_{i}^{*}$ is a flux correction from the low-order to the clipped solution. To recover mass conservation per cell we need to modify the fluxes $f_{i}^{*} \mapsto f_{i}$, so that $\sum_{i \in N_{K}} f_{i}=0, \forall K \in \mathcal{T}_{h}$. The modification of the fluxes has to be done without creating violations of the Maximum Principle; i.e., the solution must remain in bounds.

\subsection{Mass conservation via flux scaling}

To enforce local mass conservation in element $K \in \mathcal{T}_{h}$, define the local degree of freedom $U_{i}^{n+1}$ via

$$
m_{i}\left(U_{i}^{n+1}-U_{i}^{L}\right)=\alpha_{i} f_{i}^{*},
$$

where $0 \leq \alpha_{i} \leq 1$. The nodal correction factors $\alpha_{i}$ are defined so that $\sum_{i \in N_{K}} \alpha_{i} f_{i}^{*}=0$. Each factor $\alpha_{i}$ contributes to enforcing local mass conservation on the element $K$ to which the local degree of freedom $U_{i}$ belongs. Note that this is always possible. In particular, one might choose $\alpha_{i}=0$ which gives back the low-order solution. Assuming the low-order solution is conservative, i.e., $\sum_{i} m_{i} U_{i}^{L}=\sum_{i} m_{i} U_{i}^{0}$, we get

$$
\sum_{K} \sum_{i \in N_{K}} m_{i} U_{i}^{n+1}=\sum_{i} m_{i} U_{i}^{0} \Longrightarrow \int_{\Omega} u_{h}(\mathbf{x}, t) d \mathbf{x}=\int_{\Omega} u_{h}(\mathbf{x}, 0) d \mathbf{x},
$$

i.e., the method (23) is mass conservative.

Theorem 6.4.1 (Maximum-Principle Preservation (MPP)). Given $0 \leq \alpha_{i} \leq 1$ and provided $U_{i}^{*}$ and $U_{i}^{L}$ are in bounds; i.e., $U_{i}^{\min } \leq U_{i}^{* / L} \leq U_{i}^{\max }$, the method (23) is MPP; i.e., $U_{i}^{\min } \leq U_{i}^{n+1} \leq U_{i}^{\max }$, where $U_{i}^{\min }:=\min _{j \in N_{i}} U_{j}^{n}$ and $U_{i}^{\max }:=\max _{j \in N_{i}} U_{j}^{n}$.

Proof. Rewrite (23) as

$$
U_{i}^{n+1}=U_{i}^{L}+\alpha_{i} m_{i}^{-1} f_{i}^{*}=U_{i}^{L}+\alpha_{i}\left(U_{i}^{*}-U_{i}^{L}\right)=\alpha U_{i}^{*}+\left(1-\alpha_{i}\right) U_{i}^{L} .
$$

Since $0 \leq \alpha_{i} \leq 1$ and $U_{i}^{* / L} \leq U_{i}^{\max }$, we get

$$
U_{i}^{n+1} \leq \alpha_{i} U_{i}^{\max }+\left(1-\alpha_{i}\right) U_{i}^{\max }=U_{i}^{\max } \Longrightarrow U_{i}^{n+1} \leq U_{i}^{\max } .
$$

The lower bound is proven similarly.

There are different strategies to choose the interpolating parameters. A first approach, which we refer to as uniform scaling, is to scale down the dominant fluxes by the same factor. Consider a representative cell $K \in \mathcal{T}_{h}$ and define

$$
S_{K}^{+}=\sum_{\substack{f_{i}^{*}>0 \\ i \in N_{K}}} f_{i}^{*}, \quad S_{K}^{-}=\sum_{\substack{f_{i}^{*}<0 \\ i \in N_{K}}} f_{i}^{*}
$$


If $S_{K}^{+}+S_{K}^{-}>0, i \in N_{K}$, we choose

$$
\alpha_{i}:= \begin{cases}-\frac{S_{K}^{-}}{S_{K}^{+}} & \text {if } f_{i}^{*}>0 \\ 1 & \text { otherwise }\end{cases}
$$

If $S_{K}^{+}+S_{K}^{-}<0, i \in N_{K}$, we choose

$$
\alpha_{i}:= \begin{cases}1 & \text { if } f_{i}^{*} \geq 0 \\ -\frac{S_{K}^{+}}{S_{K}^{-}} & \text {otherwise }\end{cases}
$$

and if $S_{K}^{+}+S_{K}^{-}=0$ then $\alpha_{i}=1$. It is easy to see that $0 \leq \alpha_{i} \leq 1$ and $\sum_{i \in N_{K}} \alpha_{i} f_{i}^{*}=0, \forall K \in \mathcal{T}_{h}$. We refer to the combination of the DG-EFCT method and this uniform scaling approach as the DG-EFCT-U method.

Another option for choosing the interpolating parameters $\left\{\alpha_{i}\right\}$ is to solve the following minimization problem:

$$
\min _{\alpha_{i}} \sum_{i}\left(U_{i}^{n+1}-U_{i}^{H}\right)^{2}=\min _{\alpha_{i}} \sum_{i}\left(\alpha_{i} f_{i}^{*}-f_{i}^{H}\right)^{2}
$$

such that

$$
0 \leq \alpha_{i} \leq 1, \quad \sum_{i \in N_{K}} \alpha_{i} f_{i}^{*}=0
$$

for all cells $K \in \mathcal{T}_{h}$. One can find many more strategies to find the interpolating parameters $\left\{\alpha_{i}\right\}$. Bochev et al. [5] used this kind of constrained optimization in the context of conservative remapping. However, in their flux-variable flux-target (FVFT) algorithm, local mass conservation was enforced by imposing the anti-symmetry constraint on the fluxes leading to a global inequality-constrained quadratic programming problem, which has relatively high computational cost. As an alternative the authors developed a globally conservative mass-variable mass-target (MVMT) remap method which is more efficient. In contrast, in our DG method, the optimization problems for $\left\{\alpha_{i}\right\}$ are decoupled (i.e. they are local to each element) and can be solved in parallel.

In Figure 11 we show results of the non-smooth 1D problem (13) computed by the DG-EFCT-U method (24) and the minimization problem (25) with $\mathbb{Q}_{2}, \mathbb{Q}_{5}, \mathbb{Q}_{11}$ and $\mathbb{Q}_{23}$ spaces. The number of cells is adjusted to have 768 DOFs in all simulations. We can easily identify a problem, namely, the solution is more dissipated as we consider higher-order spaces. We recall from Figures 3 and 10 that the low-order method and the clipping process produce more dissipative results as we increase the order, which is part of the problem. In addition, the flux scaling used to recover mass conservation is introducing additional dissipation as the order is increased.

The recovery of mass conservation is performed cell-wise. When higher-order spaces are used, more DOFs have to be considered within a cell in order to achieve this. Therefore, locality is lost with respect to DOFs. Motivated by this, in the following section we propose a process to recover mass conservation that is more localized, that is, the distribution of mass is performed differently in different parts of a given cell. 


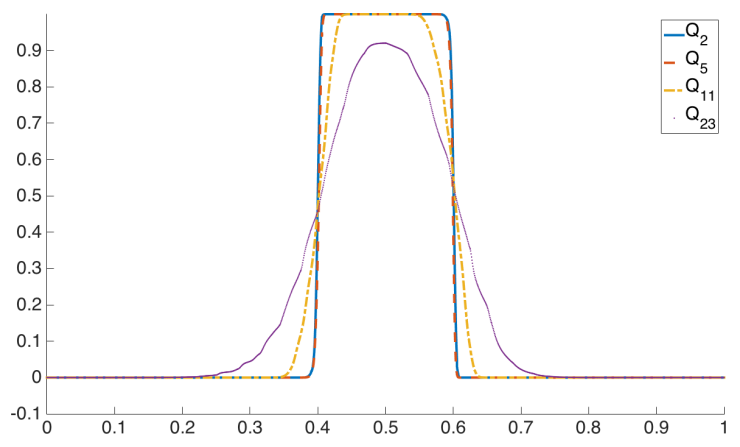

(a) Uniform scaling

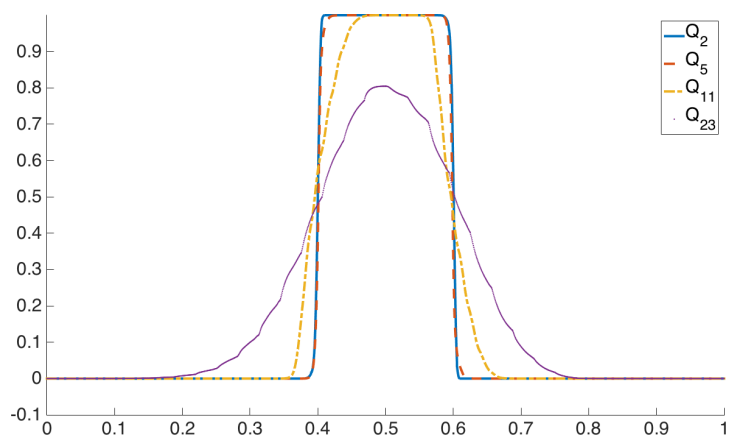

(b) L2 minimization

Figure 11: 1D simulations with non-smooth initial data via the uniform scaling (24) v.s. the L2 minimization problem (25) for the recovery of mass conservation. We consider $\mathbb{Q}_{2}, \mathbb{Q}_{5}, \mathbb{Q}_{11}$ and $\mathbb{Q}_{23}$ spaces. The number of cells is adjusted to have 768 DOFs in all simulations.

\subsection{Mass conservation via penalization}

In this section the recovery of mass conservation is localized at sub-cell level. We discuss two possible strategies.

In [7], a repair approach is used to obtain an MPP mass conservative method. This repair considers a given cell and those adjacent to it. If a criteria-satisfying solution cannot be found, more cells are considered, until a mass conservative, in bounds, solution is obtained. We can apply this idea to redistribute the mass restricted to a cell, i.e., consider a DOF within a cell and try to distribute the mass considering just adjacent DOFs in such a way that the mass for this set of DOFs equals the mass of the high-order flux $f^{H}$ on the set. If that is impossible without violating the MPP, we would consider a larger set. In the worst case scenario, we would have to consider the entire cell and use an approach similar to those presented in Section 6.4.

Another approach to distribute the mass within a cell is as follows. In [15], the author obtains a solution in bounds by clipping the solution and doing a global fix in mass using a Lagrange multiplier. We use that same idea, but restricted to a single cell. Suppose the mass error is positive in cell $K$, namely, $\delta_{K}:=\sum_{i \in N_{K}} f_{i}^{*}>0$. The final solution is based on the fluxes $\left\{\bar{f}_{i}\right\}$, which provide a mass-conservative penalization of the fluxes $\left\{f_{i}^{*}\right\}$ :

$$
m_{i}\left(U_{i}^{n+1}-U_{i}^{L}\right)=\bar{f}_{i}, \quad \text { where } \quad \bar{f}_{i}= \begin{cases}f_{i}^{*} & \text { if } f_{i}^{*} \leq 0 \\ f_{i}^{*}-\lambda_{K} w_{i} & \text { if } f_{i}^{*}>0 \text { and } f_{i}^{*}-\lambda_{K} w_{i} \geq 0 \\ 0 & \text { if } f_{i}^{*}>0 \text { and } f_{i}^{*}-\lambda_{K} w_{i}<0\end{cases}
$$

In this case (when $\delta_{K}>0$ ) negative fluxes are not penalized, but every $f_{i}^{*}>0$ is modified to $\bar{f}_{i} \in\left[0, f_{i}^{*}\right.$ ). Each quantity $w_{i}>0$ (defined later) controls the amount of penalization applied to the flux $f_{i}^{*}$. The common factor $\lambda_{K}>0$ (also defined later) is used to retain mass conservation. Before going into 
the details of $w_{i}$ and $\lambda_{K}$, we point out that the formulation (26a) is MPP. More specifically, $U_{i}^{n+1}$ is always between $U_{i}^{L}$ and $U_{i}^{*}$, which are both in bounds.

Looking at $(26 \mathrm{a})$, one can see that the choice $w_{i}=f_{i}^{*}$ reduces to the method of uniform scaling (24), where all positive fluxes are scaled with the same constant. Starting from the idea that we prefer to penalize fluxes that are different from the high-order ones, we could construct a non-uniform, localized penalization terms $w_{i}=m_{i}\left|U_{i}^{*}-U_{i}^{H}\right|$. However, this choice is too aggressive, over-correcting in a way that is not smooth. The localization can be relaxed by taking a maximum over a local neighborhood, i.e., $w_{i}=\max _{j \in N_{i}^{*}} m_{i}\left|U_{j}^{*}-U_{j}^{H}\right|$. This choice localizes penalizations only to those values which were diffused to satisfy bounds plus the immediate stencil. An important requirement is that $w_{i}>0$ whenever $f_{i}^{*}>0$, because having some $w_{i}=0$ implies that the flux $f_{i}^{*}$ is not penalized, which may lead to a situation where mass conservation cannot be restored. The final step in producing a smoother penalization is to blend the localized and uniform formulations with a tunable parameter, so that:

$$
w_{i}=(1-\theta) f_{i}^{*}+\theta \max _{j \in N_{i}^{*}} m_{j}\left|U_{j}^{*}-U_{j}^{H}\right|>0,
$$

where $\theta \in[0,1)$ and $N_{i}^{*}$ is the tighter stencil described in Figure 6 . Higher $\theta$ increases of the influence of the localized penalization; i.e., big differences with the high-order solution increase the penalization amount, which in the worst case makes $\bar{f}_{i}=0$, and thus $U^{n+1}=U^{L}$.

Having all penalization terms $w_{i}>0$, we compute $\lambda_{K}$ by solving the non-linear equation

$$
\sum_{i \in N_{K}} \bar{f}_{i}\left(\lambda_{K}\right)=0
$$

and thus enforcing mass conservation. Note that each function $\bar{f}_{i}\left(\lambda_{K}\right)$ is continuous, piecewise linear and non-increasing, hence the same holds for their sum. Furthermore,

$$
\sum_{i \in N_{K}} \bar{f}_{i}(0)=\sum_{f_{i}^{*}<0} f_{i}^{*}+\sum_{f_{i}^{*} \geq 0} f_{i}^{*}=\delta_{k}>0, \quad \sum_{i \in N_{K}} \bar{f}_{i}(+\infty)=\sum_{f_{i}^{*}<0} f_{i}^{*} \leq 0,
$$

hence (27) defines a unique $\lambda_{K}$ if at least one $f_{i}^{*}<0$, otherwise the solutions for $\lambda_{K}$ are an interval of the form $\left[\lambda^{*},+\infty\right)$. Note that by definition $w_{i}>0$ whenever $f_{i}^{*}>0$.

The case $\delta_{K}<0$ is treated similarly, so that penalization is applied to the negative fluxes:

$$
\begin{aligned}
m_{i}\left(U_{i}^{n+1}-U_{i}^{L}\right) & =\bar{f}_{i}, \quad \text { where } \quad \bar{f}_{i}= \begin{cases}f_{i}^{*} & \text { if } f_{i}^{*} \geq 0, \\
f_{i}^{*}-\lambda_{K} w_{i} & \text { if } f_{i}^{*}<0 \text { and } f_{i}^{*}-\lambda_{K} w_{i} \leq 0, \\
0 & \text { if } f_{i}^{*}<0 \text { and } f_{i}^{*}-\lambda_{K} w_{i}>0,\end{cases} \\
w_{i} & =(1-\theta) f_{i}^{*}-\theta \max _{j \in N_{i}^{*}} m_{j}\left|U_{j}^{*}-U_{j}^{H}\right|<0 .
\end{aligned}
$$

In this case equation (27) defines a unique $\lambda_{K}$, because each function $\bar{f}_{i}\left(\lambda_{K}\right)$ is continuous, piecewise linear and non-decreasing, and

$$
\sum_{i \in N_{K}} \bar{f}_{i}(0)=\sum_{f_{i}^{*}>0} f_{i}^{*}+\sum_{f_{i}^{*} \leq 0} f_{i}^{*}=\delta_{k}<0, \quad \sum_{i \in N_{K}} \bar{f}_{i}(+\infty)=\sum_{f_{i}^{*}>0} f_{i}^{*} \geq 0 .
$$


We refer to the combination of the DG-EFCT method and this non-linear penalization approach as the DG-EFCT-N method. In Figure 12, we consider the non-smooth 1D problem (13) and compare the results using the DG-EFCT-U and the DG-EFCT-N (with $\theta=0.99$ ) methods. The improvement in the solutions is clear.

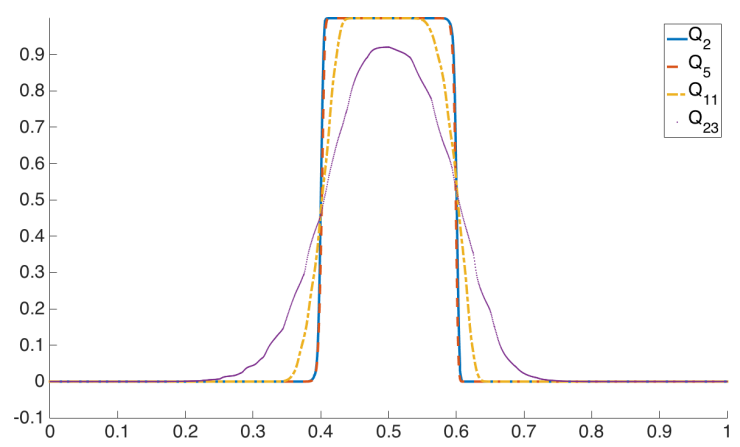

(a) Uniform scaling

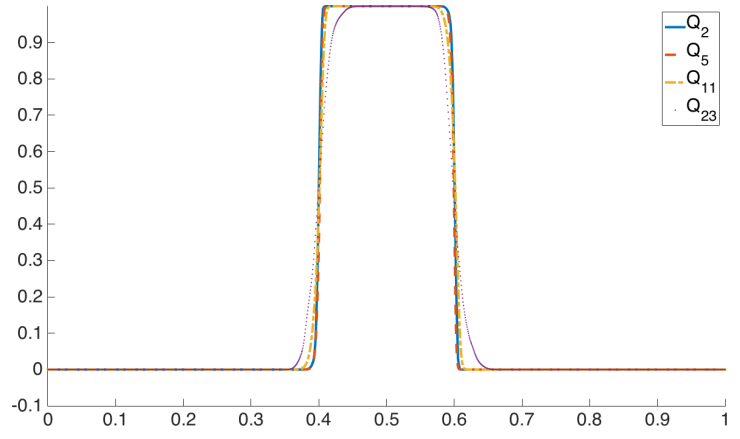

(b) Localized mass redistribution via penalization

Figure 12: 1D simulations with non-smooth initial data. Comparison between the (a) DG-EFCT-U and (b) DG-EFCT-N methods. We consider $\mathbb{Q}_{2}, \mathbb{Q}_{5}, \mathbb{Q}_{11}$ and $\mathbb{Q}_{23}$ spaces. The number of cells is adjusted to have 768 DOFs in all simulations.

\subsection{DG-EFCT Algorithm Summary}

Now we summarize the major DG-EFCT steps performed inside a single Runge-Kutta stage. Specific implementations can be derived that combine or interchange them in order to improve performance and reuse data structures.

1. Assembly of the sparse matrices $M$ and $K$, as well as computation of $m_{i}=M_{i i}^{*}$ for every index $i$.

2. Computation of $\Delta t$, so that $m_{i}+\Delta t K_{i i}^{*} \geq 0$ for every index $i$.

3. Given $U^{n},(15)$ is used to compute $U^{\min }$ and $U^{\max }$.

4. Computation of $U^{L}$ by (7) and $U^{H}$ by (6).

5. Computation of the clipped solution $U^{*}$ by (20).

6. Mass correction on each cell by (26), which includes solving equation (27).

Note that the first two steps should be performed only once, since they are independent of time. 


\section{Applicability to Advection Based Remap}

Because the original motivation behind this study was in the context of advection based finite element remap, and remap results are presented in Section 8, this section is needed to establish the connection between advection remap and the spatial discretization from Section 2.1. Detailed description of the DG advection based remap is given in [4], and only the major points are repeated here for completeness.

The goal of remap is to transfer a field $\rho$, defined on initial spatial domain $\tilde{\Omega} \subset \mathbb{R}^{d}$, to a new domain, $\Omega \subset \mathbb{R}^{d}$. For two corresponding points $\tilde{\mathbf{x}} \in \tilde{\Omega}$ and $\mathbf{x} \in \Omega$, we define a continuous transition function $F(\tilde{\mathbf{x}}, \tau): \tilde{\Omega} \times[0,1] \rightarrow \mathbb{R}^{d}$ and pseudo-velocity $\mathbf{v}(\tilde{\mathbf{x}}, \tau)$, such that

$$
F(\tilde{\mathbf{x}}, 0)=\tilde{\mathbf{x}}, \quad F(\tilde{\mathbf{x}}, 1)=\mathbf{x}, \quad \mathbf{v}(\tilde{\mathbf{x}}, \tau)=\frac{\partial F}{\partial \tau} .
$$

Here $\tau$ is pseudo-time in which the domain $\tilde{\Omega}$ transitions to $\Omega$. Then one can introduce the concept of pseudo-material derivative along the trajectories $\mathbf{x}(\tau)=F(\tilde{\mathbf{x}}, \tau)$ as

$$
\frac{d}{d \tau} \rho(x(\tau), \tau)=\frac{\partial \rho}{\partial \tau}+\mathbf{v} \cdot \nabla \rho
$$

Because the goal of remap is to preserve the initial field with respect to an Eulerian frame, i.e. $\frac{\partial \rho}{\partial \tau}=0$, one commonly used approach for remap is to define $\rho$ by solving the pseudo-time advection equation

$$
\frac{d \rho}{d \tau}=\mathbf{v} \cdot \nabla \rho
$$

Utilizing the Reynolds transport theorem, basis functions that follow the pseudo-time deformation (i.e. $\left.\frac{d \phi}{d \tau}=0\right)$ and $(29)$, one can obtain

$$
\frac{d}{d \tau} \int_{U(\tau)} \rho \phi d \mathbf{x}=-\int_{\Omega(\tau)} \rho \mathbf{v} \cdot \nabla \phi d \mathbf{x}+\int_{\partial \Omega(\tau)} \rho \mathbf{v} \cdot \mathbf{n} \phi d \mathbf{s} .
$$

Then choosing $\rho_{h} \in X_{h}$ as a finite element approximation of $\rho$, and using the Section 2.1 definitions of jump and average across faces, the semi-discrete approximation of (30) becomes

$$
\frac{d}{d \tau} \int_{\Omega} \rho_{h} \phi d \mathbf{x}=-\sum_{K \in \mathcal{T}_{h}} \int_{K} \rho_{h}(\mathbf{v} \cdot \nabla \phi) d \mathbf{x}+\sum_{f \in \mathcal{F}_{h}} \int_{f}\left\{\rho_{h} \mathbf{v} \cdot \mathbf{n}_{f}\right\}_{*}[[\phi]] d \mathbf{s} .
$$

Note that the corresponding matrix-vector form can be written in terms of the matrices $M$ and $K$ from (5), namely,

$$
\frac{d}{d \tau}(M \boldsymbol{\rho})=K \boldsymbol{\rho},
$$

where $\boldsymbol{\rho}$ is the vector of unknowns. Since the mesh $x(\tau)$ is moving, $M$ and $K$ depend on $\tau$. Therefore, the system of ordinary differential equations for $\rho$ has the form

$$
M \frac{d \boldsymbol{\rho}}{d \tau}=\left(K-\frac{d M}{d \tau}\right) \boldsymbol{\rho},
$$


with advection matrix

$$
\left(K-\frac{d M}{d \tau}\right)_{i j}=\int_{\Omega(\tau)} \phi_{i}\left(\mathbf{v} \cdot \nabla \phi_{j}\right) d \mathbf{x}-\sum_{f \in \mathcal{F}_{h}(\tau)} \int_{f}\left(\phi_{i} \mathbf{v} \cdot \mathbf{n}_{f}\right)_{d}\left[\left[\phi_{j}\right]\right] d \mathbf{s}
$$

where $\left(\phi_{i} \mathbf{v} \cdot \mathbf{n}_{f}\right)_{d}$ denotes the downwind flux:

$$
\left(\phi_{i} \mathbf{v} \cdot \mathbf{n}_{f}\right)_{d}=\left(\mathbf{v} \cdot \mathbf{n}_{f}\right)\left\{\phi_{i}\right\}+\frac{1}{2}\left|\mathbf{v} \cdot \mathbf{n}_{f}\right|\left[\left[\phi_{i}\right]\right] .
$$

It is instructive to view this advection matrix as the standard matrix $K$ plus a correction term that takes into account the mesh motion $\frac{d \mathbf{x}}{d \tau}=\mathbf{v}$. Without going into details (see Section 3 in [4]), discrete lumping of $M$ and upwinding of $K-\frac{d M}{d \tau}$ results in a conservative and monotone low-order method. Therefore, the aforementioned FCT methods can be applied to the problem (32) in order to obtain monotone and conservative high-order solution. The difference in the numerical algorithm, see Section 6.6, is that the mass and advection matrices are time dependent, and the last step of each Runge-Kutta stage becomes the mesh update $\mathbf{x}^{n+1}=\mathbf{x}^{n}+\Delta \tau \mathbf{v}^{n}$.

\section{Numerical Examples}

First we summarize some of the results that were already shown in the preceding sections:

- The positive Bernstein basis is an appealing option in the context of high-order monotone advection, see Figures 2, 3, and Table 1.

- The localized DG-FCT method (Figures 7 and 8) controls oscillations much better than the DG-FCT method (Figures 4 and 5).

- In the DG-EFCT methods with high-order spaces, the clipped solution benefits from using the localized bounds, see Figure 9.

- For high-order spaces, the DG-EFCT-N method is sharper than the DG-EFCT-U method and the minimization based approach (25), see Figures 12 and 11.

In this section we show additional results for the DG-EFCT-N method. We begin by presenting the method's convergence properties on smooth and non-smooth problems. Next, we consider 2D advection of non-trivial $\mathbb{Q}_{5}$ functions and compare the DG-FCT, localized DG-FCT, DG-EFCT-U and DG-EFCT-N methods. Finally, we show the DG-EFCT-N method's behavior in the context of $2 \mathrm{D}$ and $3 \mathrm{D}$ advection remap of complex $\mathbb{Q}_{5}$ functions.

All presented simulations use the parameter $\theta=0.99$ and finite element functions are represented in the Bernstein basis. All experiments, unless otherwise noted, are performed via a third order RungeKutta SSP method. All results are generated with the finite element methods library MFEM [1]. 


\subsection{Convergence tests}

In this section we perform a series of convergence tests on the DG-EFCT-N method.

\section{D smooth profile without local extrema}

Consider an initial condition given by

$$
u_{h}(x, y, t=0)=\tanh ((y-0.5) / 0.25)
$$

over $\Omega=(0,1) \times(0,1)$ with velocity

$$
(u, v)=(\sin (\pi x) \cos (\pi y) \sin (2 \pi t),-\cos (\pi x) \sin (\pi y) \sin (2 \pi t)) .
$$

Since the velocity field is periodic and the problem is linear the exact solution at $T=1$ coincides with the initial condition. We consider $\mathbb{Q}_{1}, \mathbb{Q}_{2}$ and $\mathbb{Q}_{3}$ spaces and show the corresponding convergence results in Table 2. We obtain the expected convergence rates.

\begin{tabular}{|c|c|c|}
\hline $\mathrm{h}$ & $L^{1}$ error & conv \\
\hline $1.25 \mathrm{E}-01$ & $6.85 \mathrm{E}-03$ & \\
\hline $6.25 \mathrm{E}-02$ & $1.77 \mathrm{E}-03$ & 1.94 \\
\hline $3.13 \mathrm{E}-02$ & $4.18 \mathrm{E}-04$ & 2.08 \\
\hline $1.56 \mathrm{E}-02$ & $1.01 \mathrm{E}-04$ & 2.05 \\
\hline
\end{tabular}

(a) $\mathbb{Q}_{1}$ space

\begin{tabular}{|c|c|c|}
\hline $\mathrm{h}$ & $L^{1}$ error & conv \\
\hline $6.25 \mathrm{E}-02$ & $5.30 \mathrm{E}-04$ & \\
\hline $3.13 \mathrm{E}-02$ & $6.18 \mathrm{E}-05$ & 3.09 \\
\hline $1.56 \mathrm{E}-02$ & $6.91 \mathrm{E}-06$ & 3.16 \\
\hline $7.81 \mathrm{E}-03$ & $7.73 \mathrm{E}-07$ & 3.15 \\
\hline
\end{tabular}

(b) $\mathbb{Q}_{2}$ space

\begin{tabular}{|c|c|c|}
\hline $\mathrm{h}$ & $L^{1}$ error & conv \\
\hline $4.17 \mathrm{E}-02$ & $5.12 \mathrm{E}-05$ & \\
\hline $2.08 \mathrm{E}-02$ & $3.66 \mathrm{E}-06$ & 3.80 \\
\hline $1.04 \mathrm{E}-02$ & $2.19 \mathrm{E}-07$ & 4.06 \\
\hline $5.21 \mathrm{E}-03$ & $1.15 \mathrm{E}-08$ & 4.25 \\
\hline
\end{tabular}

(c) $\mathbb{Q}_{3}$ space

Table 2: $L^{1}(\Omega)$-convergence using the DG-EFCT-N method with a smooth initial profile without local extrema.

\section{D non-smooth profile}

Next we consider the problem with non-smooth initial data from Section 4, equation (13). The problem is solved using the DG-EFCT-N method and, for reference, the discontinuous Galerkin discretization without limitation, i.e., the high-order method from Section 2.1. Table 3 shows convergence rates for $\mathbb{Q}_{1}, \mathbb{Q}_{2}$ and $\mathbb{Q}_{3}$ spaces. Table 4 presents a similar comparison for $\mathbb{Q}_{2}, \mathbb{Q}_{5}$ and $\mathbb{Q}_{11}$ spaces. Note that, in Table 4, the number of cells is adjusted to have the same number of DOFs in each row, allowing a fair comparison between the errors produced by the different spaces. The DG-EFCT-N method generally produces larger errors on coarser grids, which is expected, since the low order and the clipped solutions are more dissipated for higher order polynomials. For all spaces we obtain convergence rates close to the optimal rate of 1 . Up to $\mathbb{Q}_{5}$, we observe better rates as we increase the polynomial degree. 


\begin{tabular}{|c|c|c|}
\hline NDOFs & $L^{1}$ error & conv \\
\hline 64 & $6.17 \mathrm{E}-02$ & \\
\hline 128 & $3.69 \mathrm{E}-02$ & 0.74 \\
\hline 256 & $2.19 \mathrm{E}-02$ & 0.75 \\
\hline 512 & $1.30 \mathrm{E}-02$ & 0.75 \\
\hline
\end{tabular}

(a) DG with $\mathbb{Q}_{1}$

\begin{tabular}{|c|c|c|}
\hline NDOFs & $L^{1}$ error & conv \\
\hline 64 & $5.37 \mathrm{E}-02$ & \\
\hline 128 & $3.11 \mathrm{E}-02$ & 0.78 \\
\hline 256 & $1.81 \mathrm{E}-02$ & 0.77 \\
\hline 512 & $1.06 \mathrm{E}-02$ & 0.77 \\
\hline
\end{tabular}

(d) DG-EFCT-N with $\mathbb{Q}_{1}$

\begin{tabular}{|c|c|c|}
\hline NDOFs & $L^{1}$ error & conv \\
\hline 96 & $3.24 \mathrm{E}-02$ & \\
\hline 192 & $1.85 \mathrm{E}-02$ & 0.81 \\
\hline 384 & $1.05 \mathrm{E}-02$ & 0.81 \\
\hline 768 & $5.91 \mathrm{E}-03$ & 0.83 \\
\hline
\end{tabular}

(b) DG with $\mathbb{Q}_{2}$

\begin{tabular}{|c|c|c|}
\hline NDOFs & $L^{1}$ error & conv \\
\hline 96 & $3.36 \mathrm{E}-02$ & \\
\hline 192 & $1.82 \mathrm{E}-02$ & 0.88 \\
\hline 384 & $9.85 \mathrm{E}-03$ & 0.88 \\
\hline 768 & $5.38 \mathrm{E}-03$ & 0.87 \\
\hline
\end{tabular}

(e) DG-EFCT-N with $\mathbb{Q}_{2}$

\begin{tabular}{|c|c|c|}
\hline NDOFs & $L^{1}$ error & conv \\
\hline 128 & $2.24 \mathrm{E}-02$ & \\
\hline 256 & $1.22 \mathrm{E}-02$ & 0.87 \\
\hline 512 & $6.75 \mathrm{E}-03$ & 0.85 \\
\hline 1024 & $3.64 \mathrm{E}-03$ & 0.89 \\
\hline
\end{tabular}

(c) DG with $\mathbb{Q}_{3}$

\begin{tabular}{|c|c|c|}
\hline NDOFs & $L^{1}$ error & conv \\
\hline 128 & $2.73 \mathrm{E}-02$ & \\
\hline 256 & $1.38 \mathrm{E}-02$ & 0.98 \\
\hline 512 & $7.35 \mathrm{E}-03$ & 0.90 \\
\hline 1024 & $3.88 \mathrm{E}-03$ & 0.92 \\
\hline
\end{tabular}

(f) DG-EFCT-N with $\mathbb{Q}_{3}$

Table 3: $L^{1}(\Omega)$-convergence of (a)-(c) discontinuous Galerkin discretization and (d)-(f) the DG-EFCT$\mathrm{N}$ method for a non-smooth initial profile. We consider $\mathbb{Q}_{1}, \mathbb{Q}_{2}$ and $\mathbb{Q}_{3}$ spaces.

\begin{tabular}{|c|c|c|}
\hline NDOFs & $L^{1}$ error & conv \\
\hline 192 & $1.85 \mathrm{E}-02$ & \\
\hline 384 & $1.05 \mathrm{E}-02$ & 0.81 \\
\hline 768 & $5.91 \mathrm{E}-03$ & 0.83 \\
\hline 1536 & $3.32 \mathrm{E}-03$ & 0.83 \\
\hline
\end{tabular}

(a) DG with $\mathbb{Q}_{2}$

\begin{tabular}{|c|c|c|}
\hline NDOFs & $L^{1}$ error & conv \\
\hline 192 & $1.82 \mathrm{E}-02$ & \\
\hline 384 & $9.85 \mathrm{E}-03$ & 0.88 \\
\hline 768 & $5.38 \mathrm{E}-03$ & 0.87 \\
\hline 1536 & $2.95 \mathrm{E}-03$ & 0.86 \\
\hline
\end{tabular}

(d) DG-EFCT-N with $\mathbb{Q}_{2}$

\begin{tabular}{|c|c|c|}
\hline NDOFs & $L^{1}$ error & conv \\
\hline 192 & $1.24 \mathrm{E}-02$ & \\
\hline 384 & $6.70 \mathrm{E}-03$ & 0.89 \\
\hline 768 & $3.89 \mathrm{E}-03$ & 0.78 \\
\hline 1536 & $2.00 \mathrm{E}-03$ & 0.96 \\
\hline
\end{tabular}

(b) DG with $\mathbb{Q}_{5}$

\begin{tabular}{|c|c|c|}
\hline NDOFs & $L^{1}$ error & conv \\
\hline 192 & $2.25 \mathrm{E}-02$ & \\
\hline 384 & $1.07 \mathrm{E}-02$ & 1.06 \\
\hline 768 & $5.65 \mathrm{E}-03$ & 0.92 \\
\hline 1536 & $2.69 \mathrm{E}-03$ & 1.07 \\
\hline
\end{tabular}

(e) DG-EFCT-N with $\mathbb{Q}_{5}$

\begin{tabular}{|c|c|c|}
\hline NDOFs & $L^{1}$ error & conv \\
\hline 192 & $1.39 \mathrm{E}-02$ & \\
\hline 384 & $6.35 \mathrm{E}-03$ & 1.12 \\
\hline 768 & $3.50 \mathrm{E}-03$ & 0.86 \\
\hline 1536 & $1.63 \mathrm{E}-03$ & 1.09 \\
\hline
\end{tabular}

(c) DG with $\mathbb{Q}_{11}$

\begin{tabular}{|c|c|c|}
\hline NDOFs & $L^{1}$ error & conv \\
\hline 192 & $3.51 \mathrm{E}-02$ & \\
\hline 384 & $2.00 \mathrm{E}-02$ & 0.81 \\
\hline 768 & $1.08 \mathrm{E}-02$ & 0.88 \\
\hline 1536 & $6.14 \mathrm{E}-03$ & 0.81 \\
\hline
\end{tabular}

(f) DG-EFCT-N with $\mathbb{Q}_{11}$

Table 4: $L^{1}(\Omega)$-convergence of (a)-(c) discontinuous Galerkin discretization and (d)-(f) the DG-EFCT$\mathrm{N}$ method for a non-smooth initial profile. We consider $\mathbb{Q}_{2}, \mathbb{Q}_{5}$ and $\mathbb{Q}_{11}$ spaces and adjust the number of cells to have the same number of DOFs for each refinement of the convergence test. 


\section{D smooth profile with local extrema}

Finally we consider as initial condition

$$
u_{h}(x, t=0)=\cos (2 \pi(x-0.5)),
$$

over $\Omega=(0,1)$ with velocity $v=1$. We impose periodic boundary conditions and use the initial condition as exact solution at $T=1$. We use $\mathbb{Q}_{1}, \mathbb{Q}_{2}$ and $\mathbb{Q}_{3}$ to perform a convergence test via the DG-EFCT-N method and, for reference, the discontinuous Galerkin discretization from Section 2.1. The results are shown in Table 5. One can see that no better than (slightly higher than) second order is achieved. This issue is already discussed in [4], where the authors show that the dominating error is localized in the extremal regions, while high-order accuracy is obtained in the rest of the domain. Additional details about this problem can be found in [12], where it is shown that Total Variation Diminishing (TVD) methods can't achieve better than second order convergence (in the $L^{1}$ norm) around local extrema. To resolve this problem within the context of finite volumes, it is common to allow small violations on the total variation near local extrema. Popular examples are UNO [12], ENO $[13,14]$ and WENO [22] methods. In [28,30], finite volumes and discontinuous Galerkin methods are used to obtain a solution that satisfies a strict (or global) maximum principle. To achieve highorder accuracy at local extrema, the authors reconstruct a polynomial inside cells from where the bounds are computed. A parameter-free smoothness indicator based on a hierarchical slope limiter for high-order DG methods may be used as regularity criterion for deactivation of FCT corrections at smooth extrema [17].

\begin{tabular}{|c|c|c|}
\hline NDOFs & $L^{1}$ error & conv \\
\hline 64 & $1.52 \mathrm{E}-03$ & \\
\hline 128 & $3.57 \mathrm{E}-04$ & 2.08 \\
\hline 256 & $8.63 \mathrm{E}-05$ & 2.04 \\
\hline 512 & $2.12 \mathrm{E}-05$ & 2.02 \\
\hline
\end{tabular}

(a) DG with $\mathbb{Q}_{1}$

\begin{tabular}{|c|c|c|}
\hline NDOFs & $L^{1}$ error & conv \\
\hline 64 & $2.93 \mathrm{E}-03$ & \\
\hline 128 & $6.73 \mathrm{E}-04$ & 2.12 \\
\hline 256 & $1.55 \mathrm{E}-04$ & 2.12 \\
\hline 512 & $3.57 \mathrm{E}-05$ & 2.11 \\
\hline
\end{tabular}

(d) DG-EFCT-N with $\mathbb{Q}_{1}$

\begin{tabular}{|c|c|c|}
\hline NDOFs & $L^{1}$ error & conv \\
\hline 96 & $1.94 \mathrm{E}-05$ & \\
\hline 192 & $2.42 \mathrm{E}-06$ & 3.00 \\
\hline 384 & $3.02 \mathrm{E}-07$ & 3.00 \\
\hline 768 & $3.78 \mathrm{E}-08$ & 3.00 \\
\hline
\end{tabular}

(b) DG with $\mathbb{Q}_{2}$

\begin{tabular}{|c|c|c|}
\hline NDOFs & $L^{1}$ error & conv \\
\hline 96 & $3.61 \mathrm{E}-03$ & \\
\hline 192 & $6.96 \mathrm{E}-04$ & 2.37 \\
\hline 384 & $1.29 \mathrm{E}-04$ & 2.43 \\
\hline 768 & $2.33 \mathrm{E}-05$ & 2.46 \\
\hline
\end{tabular}

(e) DG-EFCT-N with $\mathbb{Q}_{2}$

\begin{tabular}{|c|c|c|}
\hline NDOFs & $L^{1}$ error & conv \\
\hline 128 & $2.40 \mathrm{E}-07$ & \\
\hline 256 & $1.50 \mathrm{E}-08$ & 4.00 \\
\hline 512 & $9.35 \mathrm{E}-10$ & 4.00 \\
\hline 1024 & $5.93 \mathrm{E}-11$ & 3.97 \\
\hline
\end{tabular}

(c) DG with $\mathbb{Q}_{3}$

\begin{tabular}{|c|c|c|}
\hline NDOFs & $L^{1}$ error & conv \\
\hline 128 & $1.99 \mathrm{E}-03$ & \\
\hline 256 & $3.82 \mathrm{E}-04$ & 2.38 \\
\hline 512 & $7.04 \mathrm{E}-05$ & 2.43 \\
\hline 1024 & $1.22 \mathrm{E}-05$ & 2.53 \\
\hline
\end{tabular}

(f) DG-EFCT-N with $\mathbb{Q}_{3}$

Table 5: $L^{1}(\Omega)$-convergence of (a)-(c) discontinuous Galerkin discretization and (d)-(f) the DG-EFCT$\mathrm{N}$ method for a smooth initial profile with local extrema. We consider $\mathbb{Q}_{1}, \mathbb{Q}_{2}$ and $\mathbb{Q}_{3}$ spaces. 


\subsection{D advection with constant velocity field}

We consider $\Omega=(0,100) \times(0,100) \subset \mathbb{R}^{2}$, velocity $\mathbf{v}=(10,10)$ and the discontinuous initial profile shown in the left panel in Figure 13. The cross shape is specified by the region $(7,32) \times(10,13) \cup$ $(14,17) \times(3,26)$ (the notation represents $\left.\left(x_{1}, x_{2}\right) \times\left(y_{1}, y_{2}\right)\right)$, which is rotated by -45 degrees around the point $(15.5,11.5)$. The lower ring's origin is at $(x, y)=(40,20)$ and its radii are 3 and 7 . The upper ring's origin is at $(x, y)=(40,40)$ and its radii are 7 and 10 . The advected function is initialized to 2 in the aforementioned subdomains, and to 0 in the rest of the domain. We compute the solution using the DG-EFCT-U method (equations (23) and (24)) and DG-EFCT-N method (equations (26) and (28)). The results are shown in Figure 13. For comparison we also show the results of the DG-FCT revisited in Section 4 and the localized DG-FCT from Section 5. For all situations we use $\mathbb{Q}_{2}$ and $\mathbb{Q}_{5}$ spaces with number of cells adjusted to have 90000 DOFs.
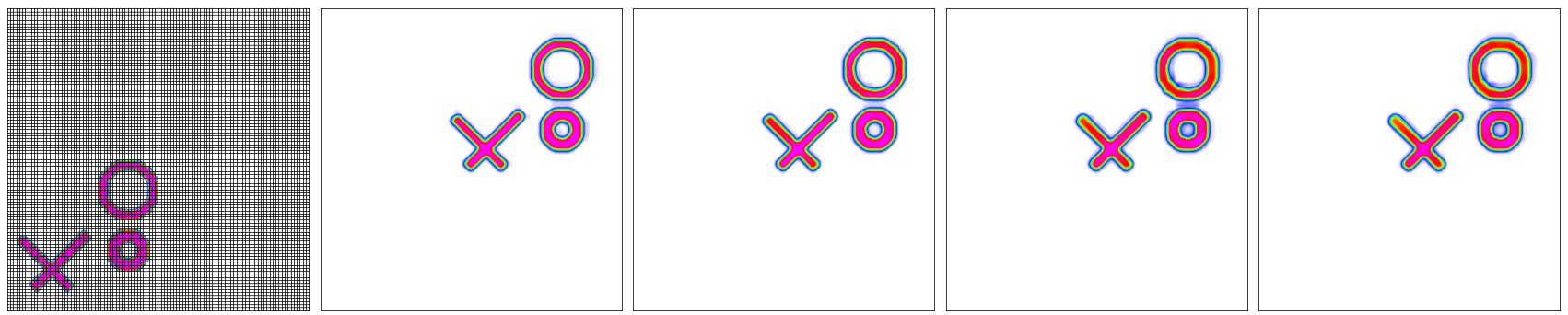

(a) $\mathbb{Q}_{2}$. From left to right: $(\min , \max )=(0,2),\left(-1.294 \times 10^{-15}, 1.99\right),\left(-2.309 \times 10^{-17}, 1.976\right),(-1.690 \times$ $\left.10^{-18}, 1.952\right)$ and $\left(-8.207 \times 10^{-17}, 1.962\right)$.
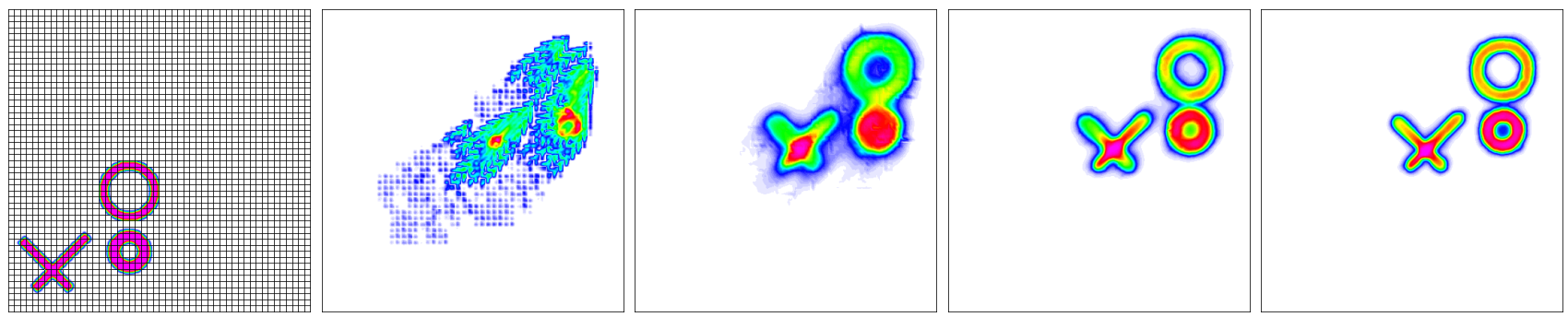

(b) $\mathbb{Q}_{5}$. From left to right: $(\min , \max )=(0,2),\left(-1.686 \times 10^{-20}, 1.322\right),\left(-6.813 \times 10^{-15}, 1.642\right),(-5.444 \times$ $\left.10^{-18}, 1.582\right)$ and $\left(-1.271 \times 10^{-16}, 1.772\right)$.

Figure 13: 2D advection with constant velocity field. We consider (a) $\mathbb{Q}_{2}$ and (b) $\mathbb{Q}_{5}$ spaces with number of cells adjusted to have 90000 DOFs in all situations. Left: initial condition with the mesh. Middle-left: solution via the DG-FCT method (11). Middle-middle: solution via the localized DG-FCT method from Section 5. Middle-right: solution via the DG-EFCT-U method (23), (24).

Right: solution via the DG-EFCT-N method (26), (28). 


\subsection{D solid body rotation on unstructured mesh}

To be consistent with the previously presented results in [4], we examine the behavior of the DGEFCT-N method for the solid body rotation benchmark. For this test problem the DG-EFCT-N method is utilized as a tool for remapping fields, as described in Section 7. Description of the initial conditions is given in [4], Section 4.3.

Lagrangian mesh motion is prescribed through the velocity field $\boldsymbol{v}(x, y)=0.1(y,-x)$. Each Lagrangian step rotates the mesh without modifying the discrete representation of the field. Each Lagrangian step is followed by a remesh / remap step where the mesh nodes are returned to their original locations and the field is remapped between the two resulting meshes. This procedure results in the field's full 360 degree rotation about the origin at final time $2 \pi / 0.1$.

Two cases are considered, each with the same total number of degrees of freedom (dof):

1. A fine mesh using $\mathbb{Q}_{2}$ discontinuous elements (9 dof/element).

2. A coarse mesh using $\mathbb{Q}_{5}$ discontinuous elements (36 dof/element).

For each case the DG-EFCT-N method is compared to the DG-FCT method used in [4]. All simulations use a fixed Lagrangian time step of size $5 \times 10^{-2}$. Each remap procedure performs five pseudo-time steps, and each pseudo-time step utilizes an explicit RK2 time integration.

Initial conditions and fields at final time for $\mathbb{Q}_{2}$ and $\mathbb{Q}_{5}$ are shown in Figure 14 and Figure 15, respectively. In Figure 14, one can see that both methods produce results of similar quality, with the DG-FCT method being slightly sharper. However, for the $\mathbb{Q}_{5}$ case, it is clear in Figure 15 that the DG-FCT method develops spurious (but monotone) oscillations that eventually distort the original shapes. The improvement in quality with the DG-EFCT-N method is clear.

\subsection{D Advection of "Balls and Jacks"}

Here we consider a full 3D version of the "balls and jacks" advection benchmark. As with the previous section, we use the DG-EFCT method as a tool for remapping fields, as described in Section 7. This non-trivial test problem addresses the case of multi-material remap.

The computational mesh consists of a box of $32^{3}$ elements in the domain $\Omega=(0,100)^{3}$. Lagrangian mesh motion is prescribed through the velocity field $v(x, y, z)=\frac{1}{\sqrt{2}}(10,10,10)$ and the problem is run to a final time of $t=6$.

We consider three non-overlapping subdomains. The subdomain $\Omega_{2}$ consists of the shape

$$
(x, y, z) \in(7,32) \times(10,13) \times(10,13) \cup(14,17) \times(3,26) \times(10,13) \cup(14,17) \times(10,13) \times(3,26),
$$

rotated by -45 degrees in the $x y$-plane, together with the shell (difference of two balls) centered at $(x, y, z)=(40,20,20)$ with radii 3 and 7 , and the shell centered at $(x, y, z)=(40,40,40)$ with radii 7 and 10. The subdomain $\Omega_{3}$ consists of the shape

$$
(x, y, z) \in(2,27) \times(30,33) \times(30,33) \cup(9,12) \times(23,46) \times(30,33) \cup(9,12) \times(30,33) \times(23,46),
$$



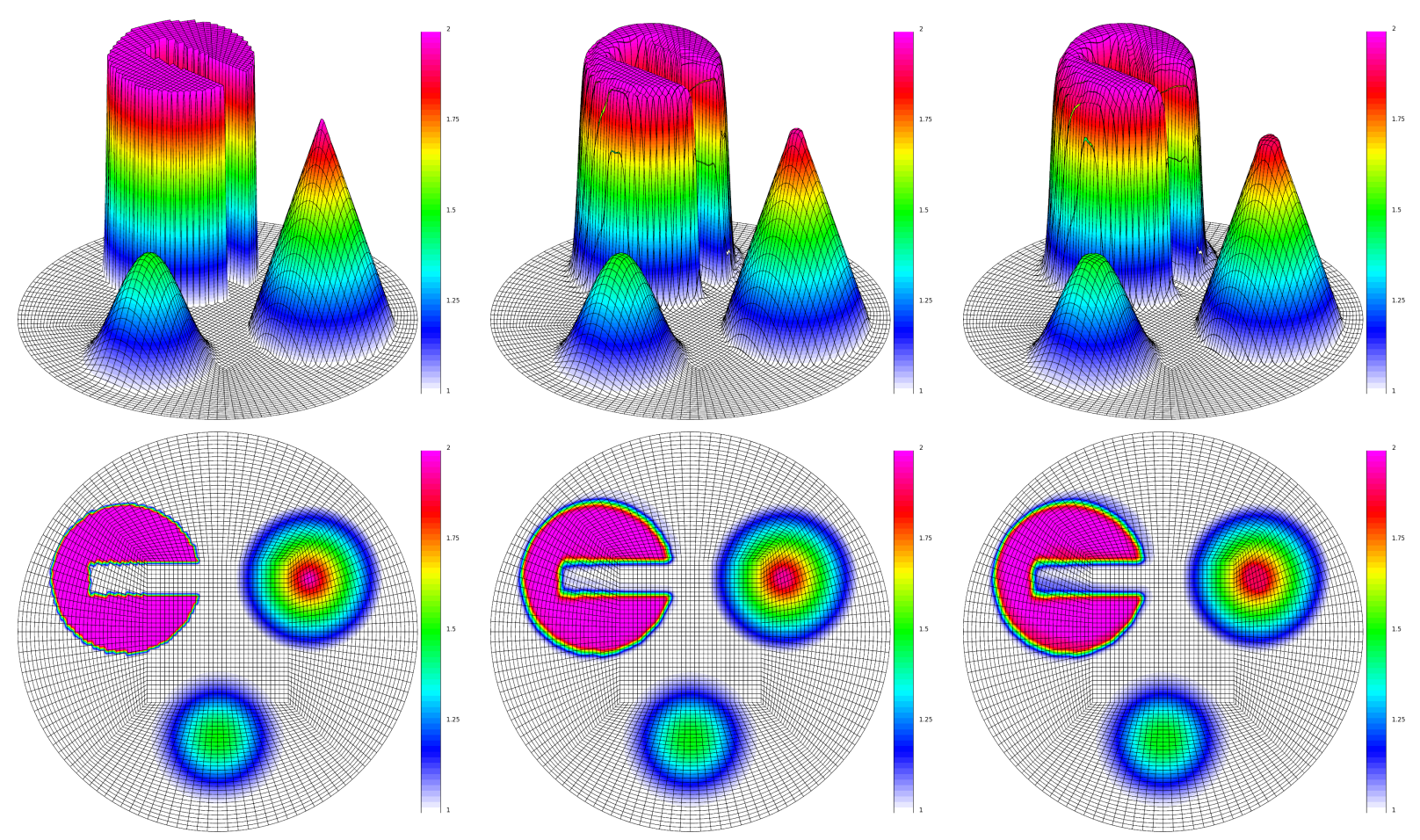

Figure 14: $3 \mathrm{D}$ view (top) and $2 \mathrm{D}$ view (bottom) of $\mathbb{Q}_{2}$ fields (left to right): initial condition, DG-FCT result, DG-EFCT-N result.

together with the ball centered at $(x, y, z)=(40,20,20)$ with radius 3 , the ball centered at $(x, y, z)=$ $(40,40,40)$ with radius 7 , and the shell centered at $(x, y, z)=(40,20,20)$ with radii 7 and 10 . The last subdomain is $\Omega_{1}=\Omega /\left(\Omega_{2} \cup \Omega_{3}\right)$. We advect three functions that correspond to these subdomains, namely,

$$
\eta_{1}(x)=\left\{\begin{array}{ll}
1 & \text { if } x \in \Omega_{1}, \\
0 & \text { otherwise }
\end{array} \quad \eta_{2}(x)=\left\{\begin{array}{ll}
1 & \text { if } x \in \Omega_{2}, \\
0 & \text { otherwise }
\end{array} \quad \eta_{3}(x)= \begin{cases}1 & \text { if } x \in \Omega_{3}, \\
0 & \text { otherwise }\end{cases}\right.\right.
$$

We use $\mathbb{Q}_{5}$ discontinuous elements which consist of 216 DoF per element in 3D. All simulations use a fixed Lagrangian time step of size $2 \times 10^{-3}$. The remap process occurs every 20 Lagrangian steps using an explicit RK2 time integration with a dynamically calculated pseudo timestep. Two cases are considered: using DG-FCT and using the new DG-EFCT-N method.

Initial conditions and results at the final time for each $\mathbb{Q}_{5}$ calculation are shown in Figure 16, which illustrates the field

$$
\eta=\eta_{1}+2 \eta_{2}+3 \eta_{3}
$$

As with the previous 2 D result for the $\mathbb{Q}_{5}$ case, it is clear in Figure 16 that the DG-FCT method develops spurious (but monotone) oscillations that completely distort the original shapes. The improvement 

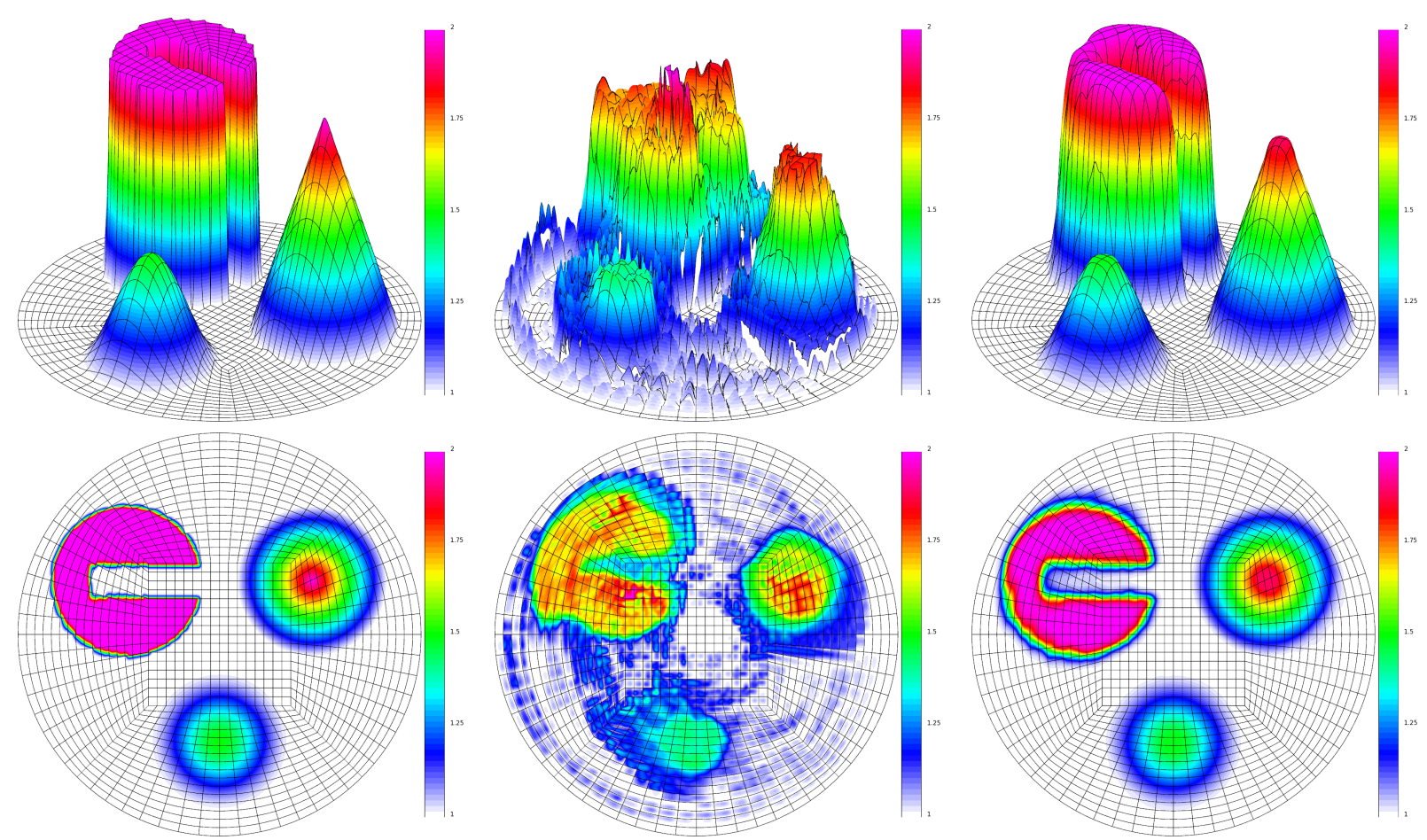

Figure 15: 3D view (top) and 2D view (bottom) of $\mathbb{Q}_{5}$ fields (left to right): initial condition, DG-FCT result, DG-EFCT-N result.

in quality with the new DG-EFCT-N method is clear.

\section{Conclusion}

We have presented a new method that addresses robustness issues with monotone advection of highorder (above $\mathbb{Q}_{3}$ ) DG finite element spaces. The DG-EFCT-N method is based on the combined effects of Bernstein polynomial basis functions, DG approximation, localized bounds, element-based flux corrections, non-linear local mass redistribution. Results have been presented for finite element spaces up to $\mathbb{Q}_{23}$ in $1 \mathrm{D}$ and $\mathbb{Q}_{5}$ in $2 \mathrm{D}$. The DG-EFCT-N method obtains optimal convergence rates for both smooth and non-smooth fields, produces maximum principle preserving solutions and highly reduces spurious oscillations; indeed, in the numerical experiments we performed we didn't observe oscillatory behavior.

A future area of research will be to fully incorporate the new method in the context of high-order curvilinear ALE hydrodynamics, where the goal is to remap composite fields in a synchronized way, e.g., remap of mass while preserving bounds for density. We also plan to extend the presented methods with interface sharpening techniques. 

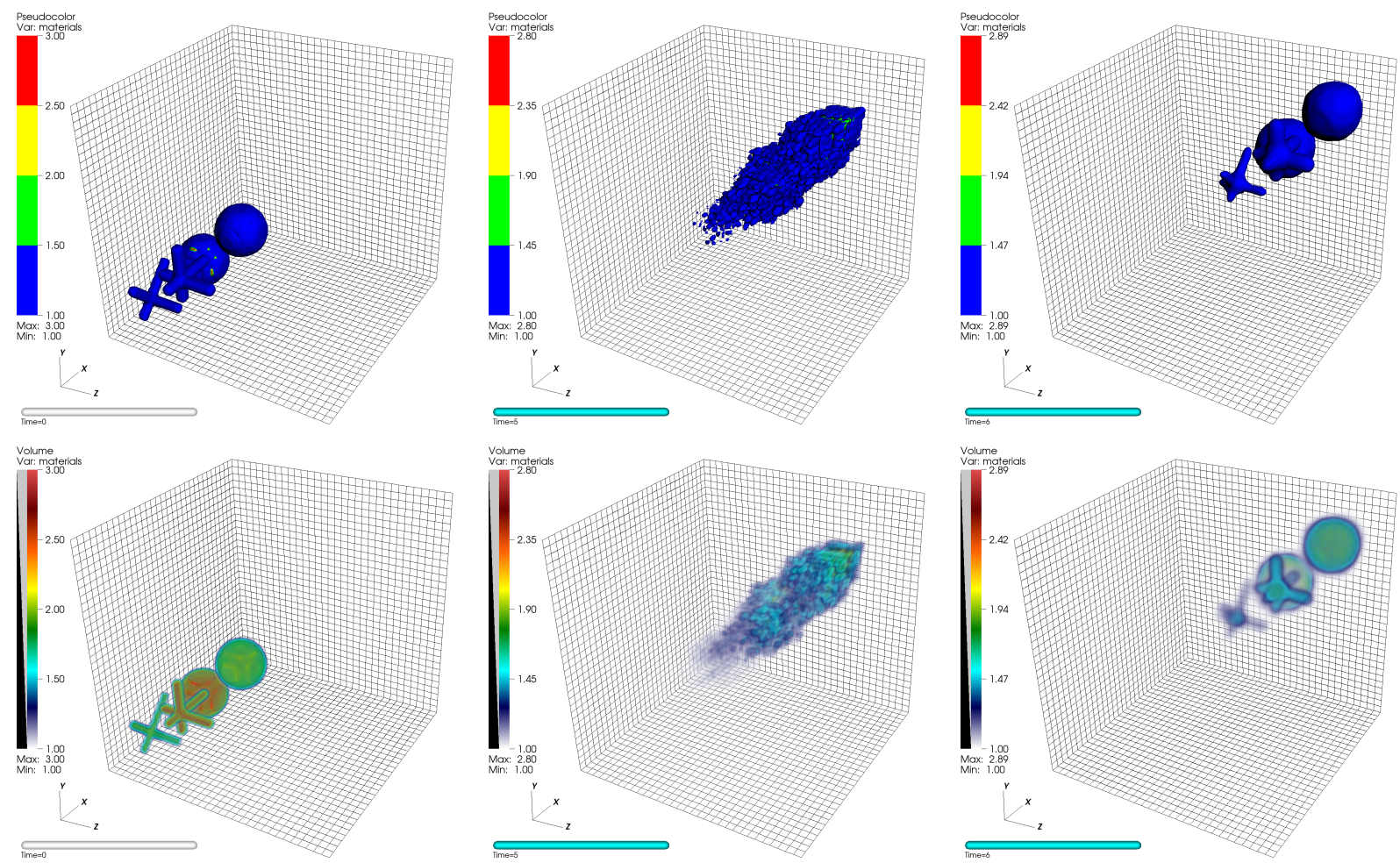

Figure 16: Iso-surface plot (top) and 3D volume rendering with transparency (bottom) of $\mathbb{Q}_{5}$ fields (left to right): initial condition, DG-FCT result, DG-EFCT-N result.

\section{Acknowledgments}

This work performed under the auspices of the U.S. Department of Energy by Lawrence Livermore National Laboratory under Contract DE-AC52-07NA27344, LLNL-JRNL-684083. The work of D. Kuzmin was supported by the German Research Association (DFG) under grant KU 1530/15-1.

\section{References}

[1] MFEM: Modular finite element methods. mfem.org.

[2] R. Abgrall and J. Trefilík. An example of high order residual distribution scheme using nonLagrange elements. Journal of Scientific Computing, 45(1):3-25, 2010.

[3] Mark Ainsworth, Gaelle Andriamaro, and Oleg Davydov. Bernstein-Bézier finite elements of arbitrary order and optimal assembly procedures. SIAM Journal on Scientific Computing, 33(6):30873109, 2011. 
[4] Robert W Anderson, Veselin A Dobrev, Tzanio V Kolev, and Robert N Rieben. Monotonicity in high-order curvilinear finite element arbitrary Lagrangian-Eulerian remap. International Journal for Numerical Methods in Fluids, 77(5):249-273, 2015.

[5] Pavel Bochev, Denis Ridzal, and Mikhail Shashkov. Fast optimization-based conservative remap of scalar fields through aggregate mass transfer. Journal of Computational Physics, 246:37-57, 2013 .

[6] Jay P Boris and David L Book. Flux-corrected transport. I. SHASTA, a fluid transport algorithm that works. Journal of Computational Physics, 11(1):38-69, 1973.

[7] Rao Garimella, Milan Kuchařík, and Mikhail Shashkov. Efficient algorithm for local-boundpreserving remapping in ALE methods. In Numerical Mathematics and Advanced Applications, pages 358-367. Springer, 2004.

[8] Sigal Gottlieb, Chi-Wang Shu, and Eitan Tadmor. Strong stability-preserving high-order time discretization methods. SIAM review, 43(1):89-112, 2001.

[9] Jean-Luc Guermond, Murtazo Nazarov, Bojan Popov, and Yong Yang. A second-order maximum principle preserving lagrange finite element technique for nonlinear scalar conservation equations. SIAM Journal on Numerical Analysis, 52(4):2163-2182, 2014.

[10] A Harten and G Zwas. Self-adjusting hybrid schemes for shock computations. Journal of Computational Physics, 9(3):568-583, 1972.

[11] Ami Harten. High resolution schemes for hyperbolic conservation laws. Journal of Computational Physics, 49(3):357-393, 1983.

[12] Ami Harten and Stanley Osher. Uniformly high-order accurate nonoscillatory schemes. i. SIAM Journal on Numerical Analysis, 24(2):279-309, 1987.

[13] Ami Harten, Stanley Osher, Björn Engquist, and Sukumar R Chakravarthy. Some results on uniformly high-order accurate essentially nonoscillatory schemes. Applied Numerical Mathematics, $2(3): 347-377,1986$.

[14] Ami Harten, Stanley Osher, Björn Engquist, and Sukumar R Chakravarthy. Uniformly high order accurate essentially non-oscillatory schemes, III. Journal of Computational Physics, 71(2):231303, 1987.

[15] Dmitri Kuzmin. A high-resolution finite element scheme for convection-dominated transport. Communications in numerical methods in engineering, 16(3):215-223, 2000.

[16] Dmitri Kuzmin, Rainald Löhner, and Stefan Turek. Flux-Corrected Transport: Principles, Algorithms, and Applications. Scientific Computation. Springer, 2005. 
[17] Dmitri Kuzmin and Friedhelm Schieweck. A parameter-free smoothness indicator for highresolution finite element schemes. Central European Journal of Mathematics, 11(8):1478-1488, 2013.

[18] Dmitri Kuzmin and Stefan Turek. Flux correction tools for finite elements. Journal of Computational Physics, 175(2):525-558, 2002.

[19] Dmitri Kuzmin and Stefan Turek. High-resolution FEM-TVD schemes based on a fully multidimensional flux limiter. Journal of Computational Physics, 198(1):131-158, 2004.

[20] Pierre Lesaint and Pierre-Arnaud Raviart. On a finite element method for solving the neutron transport equation. In C. de Boor, editor, Mathematical Aspects of Finite elements in Partial Differential Equations, pages 89-123. Academic Press, New York, 1974.

[21] Chao Liang and Zhengfu Xu. Parametrized maximum principle preserving flux limiters for high order schemes solving multi-dimensional scalar hyperbolic conservation laws. Journal of Scientific Computing, 58(1):41-60, 2014.

[22] Xu-Dong Liu, Stanley Osher, and Tony Chan. Weighted essentially non-oscillatory schemes. Journal of Computational Physics, 115(1):200-212, 1994.

[23] William H Reed and TR Hill. Triangular mesh methods for the neutron transport equation. Los Alamos Report LA-UR-73-479, 1973.

[24] Tao Xiong, Jing-Mei Qiu, and Zhengfu Xu. High order maximum-principle-preserving discontinuous galerkin method for convection-diffusion equations. SIAM Journal on Scientific Computing, 37(2):A583-A608, 2015.

[25] Zhengfu Xu. Parametrized maximum principle preserving flux limiters for high order schemes solving hyperbolic conservation laws: one-dimensional scalar problem. Mathematics of Computation, 83(289):2213-2238, 2014.

[26] Pei Yang, Tao Xiong, Jing-Mei Qiu, and Zhengfu Xu. High order maximum principle preserving finite volume method for convection dominated problems. Journal of Scientific Computing, 67(2):795-820, 2016.

[27] Steven T Zalesak. Fully multidimensional flux-corrected transport algorithms for fluids. Journal of Computational Physics, 31(3):335-362, 1979.

[28] Xiangxiong Zhang and Chi-Wang Shu. On maximum-principle-satisfying high order schemes for scalar conservation laws. Journal of Computational Physics, 229(9):3091-3120, 2010.

[29] Xiangxiong Zhang and Chi-Wang Shu. On positivity-preserving high order discontinuous galerkin schemes for compressible euler equations on rectangular meshes. Journal of Computational Physics, 229(23):8918-8934, 2010. 
[30] Xiangxiong Zhang, Yinhua Xia, and Chi-Wang Shu. Maximum-principle-satisfying and positivitypreserving high order discontinuous Galerkin schemes for conservation laws on triangular meshes. Journal of Scientific Computing, 50(1):29-62, 2012.

[31] Yifan Zhang, Xiangxiong Zhang, and Chi-Wang Shu. Maximum-principle-satisfying second order discontinuous galerkin schemes for convection-diffusion equations on triangular meshes. Journal of Computational Physics, 234:295-316, 2013. 